\title{
Attitudes, croyances et pratiques des leaders d'opinion clés (loc) et des prestataires sur la contraception d'urgence (CU) au Sénégal
}

\author{
Babacar Mane \\ Population Council \\ Saumya RamaRao \\ Population Council \\ Martha Brady \\ Population Council \\ Fatou Bintou Mbow \\ Ababacar Thiam \\ Population Council
}

Follow this and additional works at: https://knowledgecommons.popcouncil.org/departments_sbsr-rh

Part of the Health Policy Commons, International Public Health Commons, Obstetrics and Gynecology Commons, Public Health Education and Promotion Commons, and the Women's Health Commons How does access to this work benefit you? Let us know!

\section{Recommended Citation}

Mane, Babacar, Saumya RamaRao, Martha Brady, Fatou Bintou Mbow, and Ababacar Thiam. 2012.

"Attitudes, croyances et pratiques des leaders d'opinion clés (loc) et des prestataires sur la contraception d'urgence (CU) au Sénégal." Dakar: Population Council. 


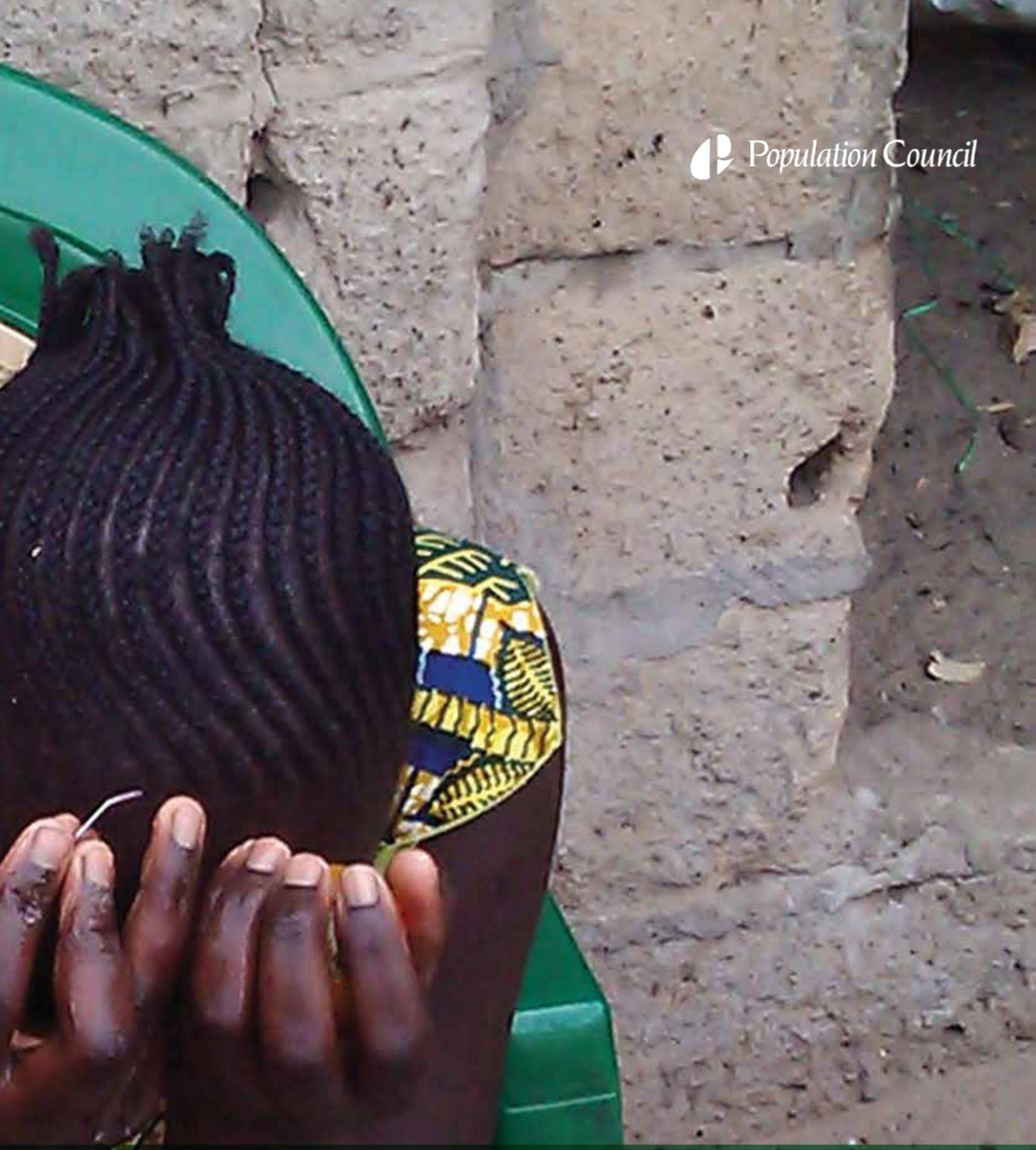

ATTITUDES, CROYANCES ET PRATIQUES DES LEADERS D’OPINION CLÉS (LOC) ET DES PRESTATAIRES SUR LA CONTRACEPTION D'URGENCE (CU) AU SÉNÉGAL

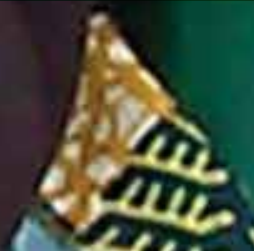




\section{OÜ TRAVAILLONS-NOUS?}

LE POPULATION COUNCIL A DES BUREAUX DANS 16 PAYS.

NOUS FAISONS DE LA RECHERCHE, CRÉONS DES PROGRAMMES ET RENFORÇONS LES COMPÉTENCES DES CHERCHEURS LOCAUX AUTOUR DU MONDE.

LES NOMS EN GRAS INDIQUENT LES PAYS DANS LESQUELS POPULATION COUNCIL A UN BUREAU.

$\triangle$ INDIQUENT DES TRAVAUX DE RECHERCHE SUR LE VIH ET LE SIDA.

INDIQUENT DES TRAVAUX DE RECHERCHE SUR LA PAUVRETÉ, LE GENRE ET LA JEUNESSE.

— INDIQUENT DES TRAVAUX DE RECHERCHE SUR LA SANTÉ DE LA REPRODUCTION.

\section{$\square$ Écosse $\succ$ \\ $\triangle$ Angleterre \\ $\Delta$ France}

$\triangle$ États-Unis

$\triangle$ Mexique

\section{Guatemala}

\section{Haïti}

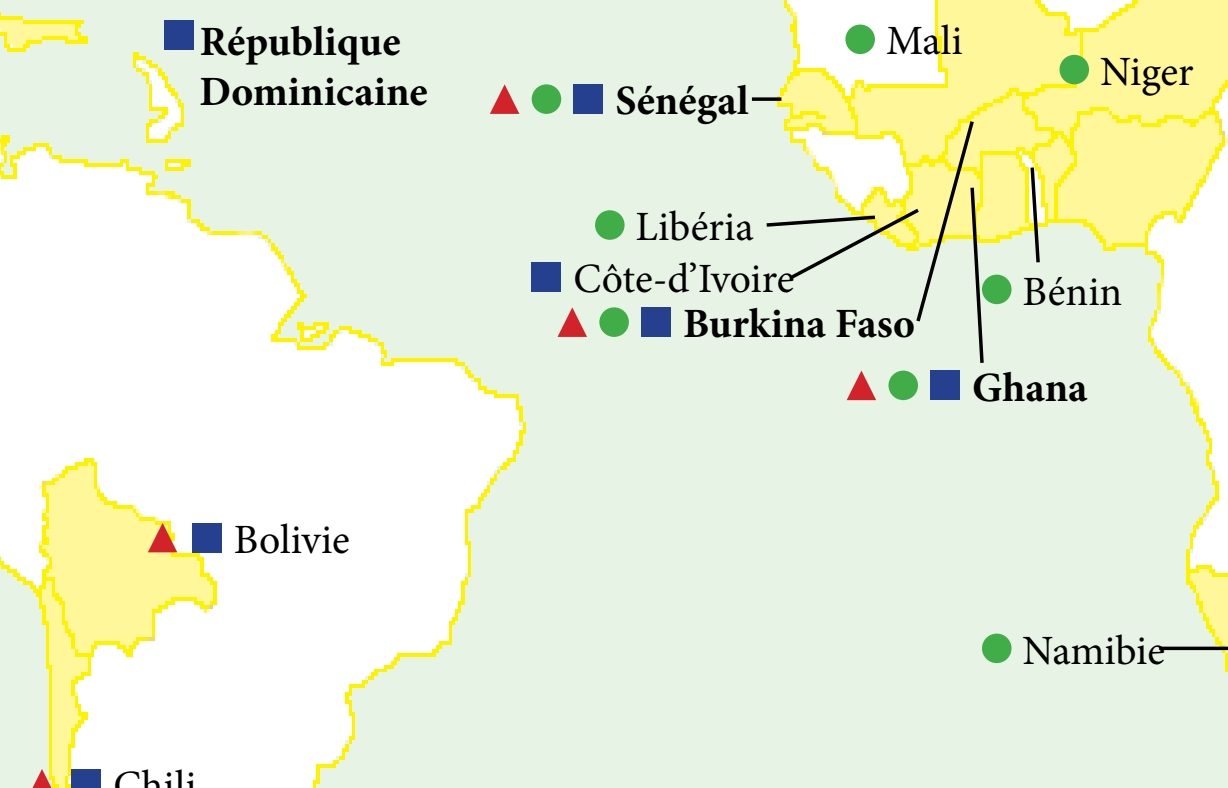


$\longrightarrow$ Syrie

$\triangle \bigcirc$ Pakistan

\section{Egypte}

Soudan

$\triangle \bigcirc$ Ethiopie

$\longrightarrow$ Ouganda

$\triangle O$ Kenya

$\Delta \bigcirc$ Zambie $\triangle \bigcirc$ Tanzanie

$\triangle$ Malawi

$\triangle$ Zimbabwe

1 Lesotho

$\triangle$ Afrique du Sud
Chine

口 Japon

Népal

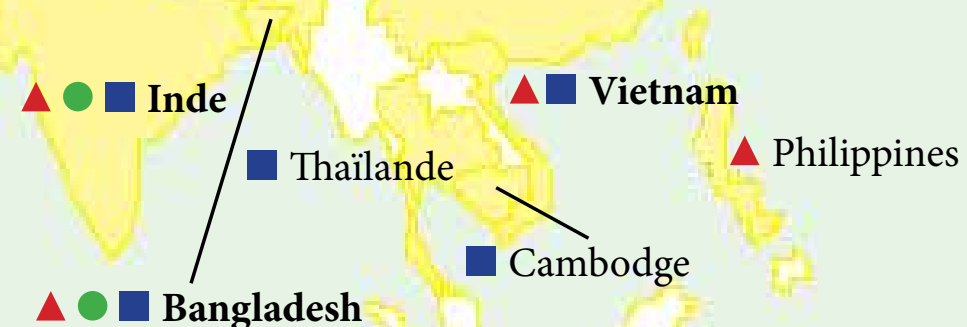

Australie 


\section{(2) Population Council}

\section{ATTITUDES, CROYANCES ET PRATIQUES \\ DES LEADERS D'OPINION CLÉS (LOC) ET DES PRESTATAIRES \\ SUR LA CONTRACEPTION D’URGENCE (CU) AU SÉNÉGAL}

Babacar Mané

Saumya Ramarao

Martha Brady

Fatou Bintou Mbow

Ababacar Thiam

Avril, 2012 


\section{Table des Matières}

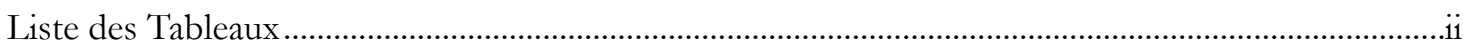

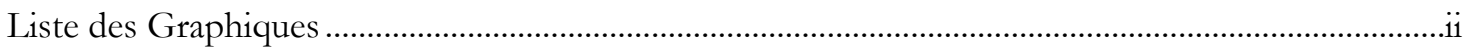

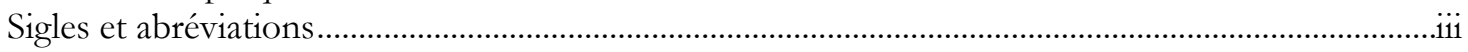

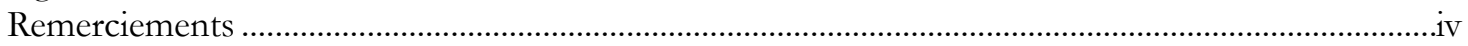

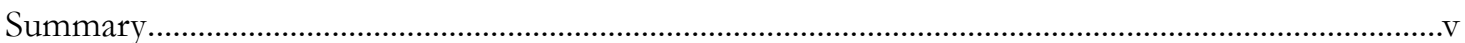

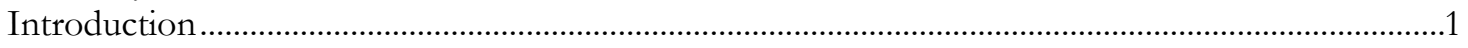

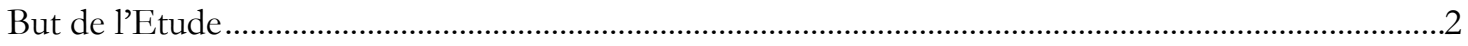

Objectifs de l'Etude

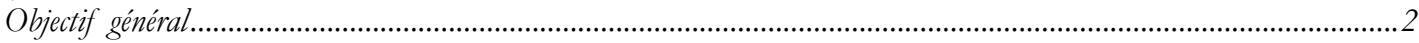

Objectifs spécifiques ..............................................................................................................................................

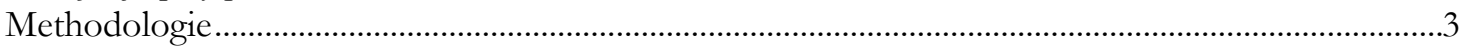

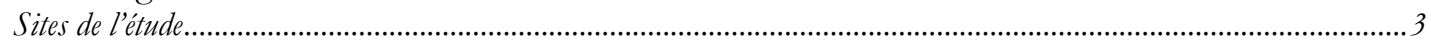

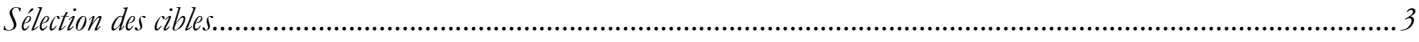

Instruments de collecte et questions explorées........................................................................................................

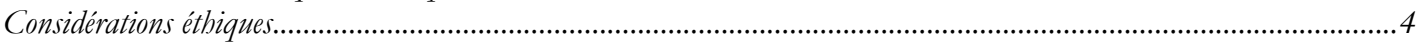

Gestion et analyse des données............................................................................................................................

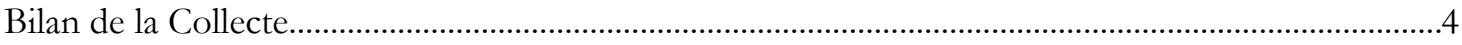

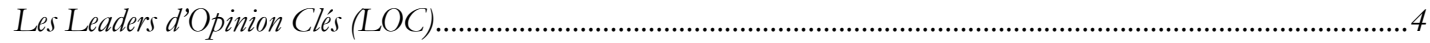

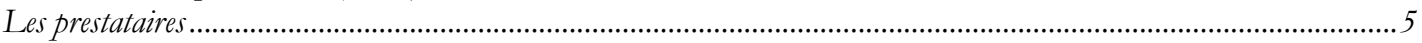

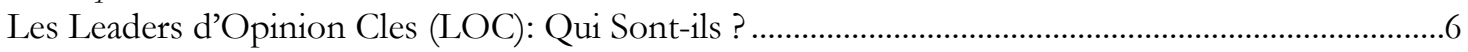

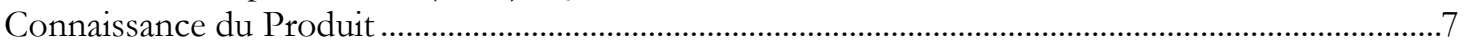

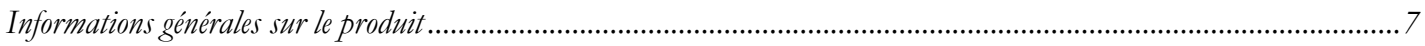

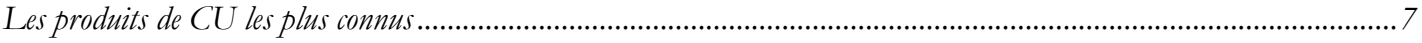

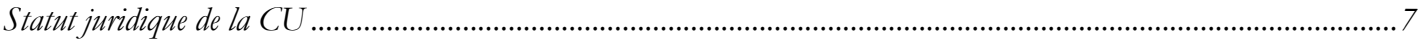

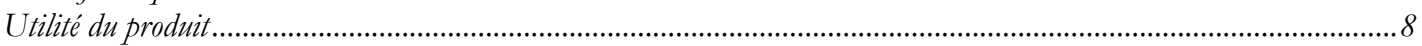

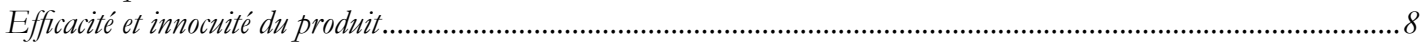

La CU dans les Politiques/ normes et directives nationales...................................................................................... 8

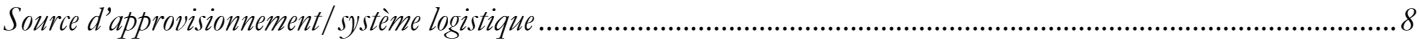

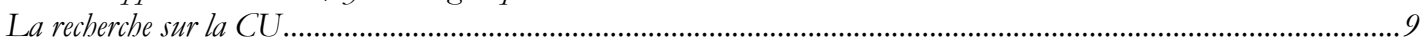

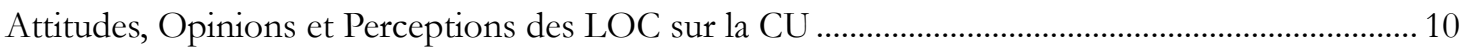

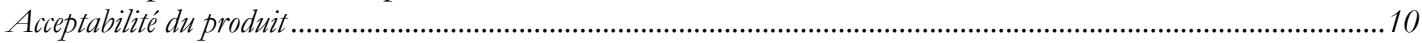

La CU peut contribuer au repositionnement de la PF....................................................................................... 10

Perceptions sur les attitudes des prestataires/ acceptabilité du produit par les prestataires................................................10

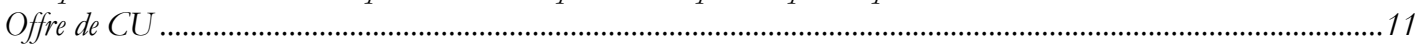

Utilisation répétée de la CU ...........................................................................................................................11

Obstacles par Rapport a l'Acces et a l'Offre de CU................................................................................ 11

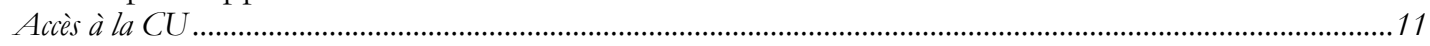

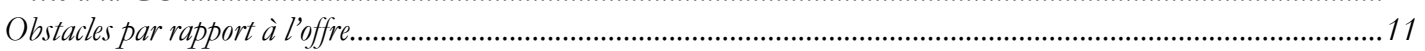

Recommandations des LOC pour la Promotion de la CU................................................................. 12

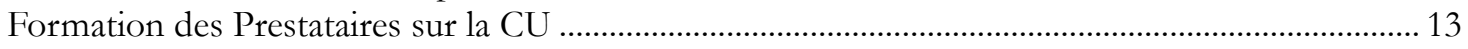

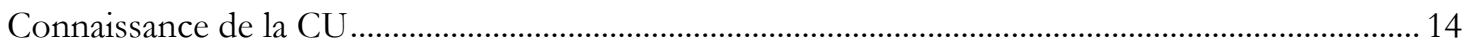

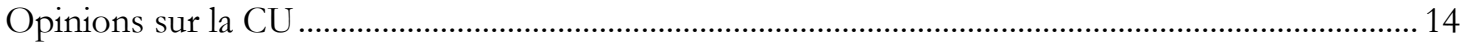

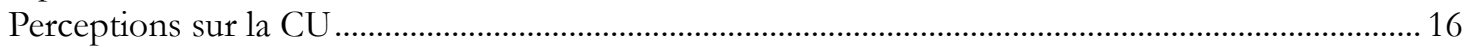

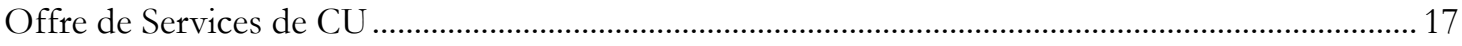

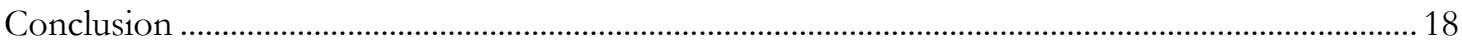

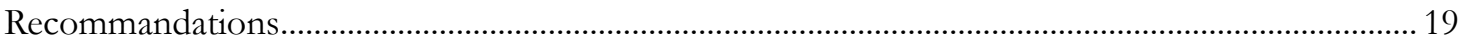

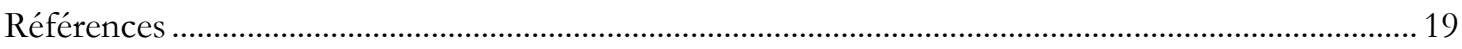




\section{Liste des Tableaux}

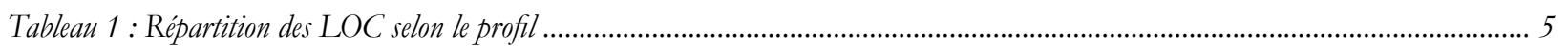

Tableau 2 : Répartition des Points de Prestations de services selon le type …..................................................................................... 5

Tableau 3 : Répartition des prestataires selon la catégorie professionnelle ….................................................................................... 5

Tableau 4 : Répartition des prestataires selon le sexe …….............................................................................................................. 5

Tableau 5 : Répartition des prestataires selon l'âge et l'expérience en matière de CU ….............................................................................. 6

Tableau $6:$ Pourcentage de prestataires offrant actuellement la CU selon la catégorie professionnelle et la région ........................................... 6

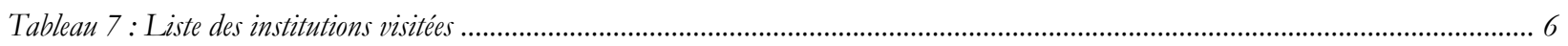

Tableau 8 : Pourcentage de prestataires ayant reçu une formation en $C U$.......................................................................................... 13

Tableau 9 : Pourcentage de prestataires ayant reçu une formation en CU selon la catégorie professionnelle ................................................. 13

Tableau 10 : Pourcentage de prestataires connaissant les modes d'action de la CU .............................................................................. 14

Tableau 11 : Pourcentage de prestataires pensant que la CU est une forme d'avortement ........................................................................ 14

Tableau 12 : Répartition des prestataires selon leurs opinions sur les endroits où la CU devrait être offerte ............................................... 16

Tableau 13 : Répartition des prestataires selon leurs opinions sur l'áge des utilisateurs de la CU ............................................................. 16

Tableau 14 : Répartition des prestataires selon leurs opinions sur le nombre de fois qu'une femme peut utiliser la CU au cours de l'année ........ 17

Tableau 15 : Répartition des prestataires selon leurs perceptions des femmes qui utilisent la CU.......................................................... 17

Tableau 16 : Répartition des prestataires selon les services habituellement offerts aux clients de la CU..................................................... 17

\section{Liste des Graphiques}

Graphique 1: Proportion de prestataires ayant déjà reçu une formation en counseling PF dans le passé selon la qualification ................... 13

Graphique 2 : Connaissances des contres indications médicales pour l'utilisation de la CU .............................................................. 14

Graphique 3 : Répartition des prestataires selon la connaissance des critères d'éligibilité pour la CU .................................................. 15

Graphique 4: Répartition des prestataires selon leur opinions sur certaines conditions de l'offre de la CU .............................................. 15

Graphique 5: Répartition des prestataires selon leur opinions sur le type de prestataire autorisé à offrir la CU ....................................... 15

Graphique 6 : Répartition des prestataires selon leurs opinions sur les cas où la $C U$ devrait être utilisée ............................................. 16

Graphique 7: Types de produits de CU habituellement offerts aux utilisateurs ............................................................................... 17

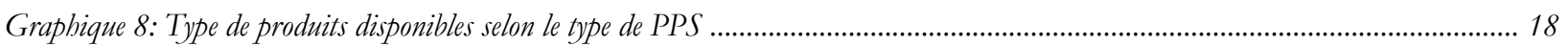




\section{Sigles et Abréviations}

ACDEV Action pour le développement

ASBEF Association pour le Bien Etre Familial

CCA Centre Conseil pour Adolescents

CEFOREP Centre de Formation et de Recherche en santé de la Reproduction

COUD Centre des Euvres Universitaires de Dakar

CU Contraception d'Urgence

DSR Division de la santé de la Reproduction

IEC Information Education Communication

ISSU Initiative sénégalaise de santé Urbaine

LOC Leaders d'Opinion Clés

OCB Organisation Communautaire de Base

OIP Offre Initiale de Pilule

ONG Organisation Non gouvernementale

PCU Pilule Contraceptive d'urgence

PF Planification Familiale

PNA Pharmacie Nationale d'Approvisionnement

PPS Point de Prestation de Services 


\section{Remerciements}

Population Council adresse ses vifs remerciements à tous ceux qui ont contribué à la réalisation de cette étude.

Nos remerciements vont à l'endroit de nos partenaires du Ministère de la Santé et de l'Action Sociale, notamment la Division de la Santé de la Reproduction (DSR) et la Division des Etudes et de la Recherche (DER) qui ont facilité la mise en œuvre de la collecte des données sur le terrain.

Nous exprimons notre profonde gratitude à l'endroit des responsables des institutions que nous avons visitées, notamment les médecins chefs de région et de district au niveau des régions de Dakar, Thiès et Mbour, les responsables des cliniques et officines privées visitées, des ONGS (ASBEF, FAWE, ACDEV, réseau Siggil Jiggen...) les responsables d'institutions nationales ( Parlement, Ministère de l'Education Nationale à travers la Division du Contrôle Médicale scolaire, le Ministère de la Jeunesse à travers le Projet Promotion des Jeunes...), les partenaires au développement (USAID, UNFPA, OMS...), les membres des réseaux professionnelles (Association des Femmes Juristes, Réseau Islam et Population, Réseau des Journalistes, Ordre des Pharmaciens, Ordre des Médecins...), les membres des Organisations Communautaires de Base (Mouvement d'Action pour les Jeunes, Synergie Banlieue...) pour leur disponibilité et leur contribution positive au cours des entretiens.

Nous adressons ainsi nos vifs remerciements à toutes ces personnes qui ont bien voulu prendre de leur temps et se prêter à nos interviews.

Nous saluons ici le professionnalisme des enquêtrices et des agents de saisie qui ont su faire preuve d'un engagement sans faille pour la réussite de l'étude.

Cette étude a pu se réaliser grâce au financement de la Fondation Bill et Melinda Gates.

Que toutes ces institutions et ces personnes qui ont ainsi pu apporter leur précieuse contribution à la réalisation de cette étude trouvent ici l'expression de notre sincère reconnaissance.

\section{Dr Nafissatou DIOP}

Population Council Sénégal

Country Director 


\section{Summary}

More often known as the "morning after" pill, the Emergency Contraceptive Pill (ECP) has been used for over 30 years throughout the world as a method for preventing unwanted pregnancy. EC is a unique contraceptive preventing unwanted pregnancy after unprotected or poorly protected sexual intercourse.

Access to EC is an important part of overall family planning (FP) strategies, especially in contexts where the magnitude of unwanted pregnancies, violence, sexual abuse, and their consequences (unsafe abortions, infanticide) is high. The issue calls for greater attention by national stakeholders.

In Senegal, initiatives for mainstreaming EC in the FP program are fairly recent. EC was included in Senegal's FP program in the mid 2000s, and by 2007-2008 it was included in the FP program's policies and guidelines. Service statistics indicate, however, that ECP distribution through the public sector is very low $(<2 \%)$. While most users access ECPs through the private sector, the volume of sales is not clear, due to the absence of an operational information system of EC distribution through pharmacies.

In an effort to strengthen EC provision in Senegal and reposition EC within the FP program, Population Council conducted a study in collaboration with the Reproductive Health Division (RHD) of the Ministry of Health, Senegal to document the positions and perceptions of Key Opinion Leaders (KOLs) who can shape the policy and program framework ; as well as documenting providers' attitudes, beliefs, and practices regarding EC. Support for this study was provided by the International Consortium for Emergency Contraception, as part of a three-country project also including Nigeria and India.

\section{Study Objectives}

\section{Global objective}

The study's overall objective is collecting information from KOLs and providers about their EC knowledge, attitudes, and practices, and understanding their options and perceptions that may influence EC policies and programs.

\section{Specific objectives}

- Documenting providers' EC attitudes, beliefs, and practices, including the issue of repeat use ;

- Capturing KOLs' views and perceptions about EC policy and program issues ;

- Collecting suggestions and recommendations for establishing appropriate interventions for repositioning $\mathrm{EC}$ in public and private health sectors.

\section{Methodology}

\section{Study sites}

The study was conducted in August and September 2011 in Dakar and Thiès urban areas targeted by the "Urban Reproductive Health Initiative in Senegal" (ISSU) project, funded by the Bill and Melinda Gates Foundation.

\section{Respondents}

The study collected information from two types of respondents: KOLs and EC providers, with 34 KOLs and 155 providers interviewed. Targeted KOLs included public authorities, parliament members, professional association members, development partners, NGO representatives, religious leaders, pharmaceutical distributors, leaders of community-based organizations, and private practitioners. Providers included a wide range of cadres and included those currently providing, or have provided, EC, both in the public and private sectors, including doctors, midwives, nurses/ nurse assistants, pharmacists, and pharmacy counter staff, working in a number of settings: public facilities, private clinics, NGO facilities, pharmacies, adolescent counseling centers, and school infirmaries. 


\section{Primary Results}

\section{Provider survey}

A considerable proportion of providers (38\%) have not received any EC training. Among those trained, about half were trained as part of on-the-job training. Nearly a third of providers were never trained in FP counseling, and nearly four out of 10 providers have never received specific training on EC provision. Staff in private pharmacies, especially counter staff, tends to be less trained.

Providers have some ECP knowledge, but it can be strengthened. For example, improvements in knowledge about ECP's mode of action, its timing of use, and eligibility criteria can be strengthened. Although many providers think ECP is a safe product, negative views persist.

- While 72 percent of providers indicate ECPs can be provided to all women, irrespective of age or marital status, when questioned in-depth, responses are lower. Less than half of providers $(43 \%)$ responded that all married women could receive ECPs, and the proportion drops for youth and adolescent girls (37\%) ;

- Forty-one percent of trained providers were comfortable with ECPs sold without prescription, while the majority preferred tighter controls;

- Most providers preferred EC provision limited to conventional health facilities such as hospitals, health centers, private clinics, and pharmacies: They do not favor extending EC at community and school levels through other supply means ;

- Opinions are mixed on EC use by married women, unmarried adolescents, and sex workers.

Analysis of providers' ECP perceptions indicates:

- Providers believe primary users of EC are aged 15 to 29 years, particularly between 20 and 29 ;

- Providers do not favor regular EC use ;

- Providers feel EC users, more than others, are at greater risk of acquiring STIs, have multiple sexual partners, and not use regular contraception;

- Over two-thirds of providers indicated providing instructions on ECP use, and nearly twofifths reported answering client questions, but fewer providers referred clients to regular FP services $(28 \%)$ or provided information that ECPs do not protect against HIV and STIs ;

- $\quad$ Norlevo ${ }^{\circledR}$ is the commonly prescribed ECP.

\section{Key opinion leader survey}

Product beliefs:

- Apart from KOLs directly involved in the managing the reproductive health program, others, such as parliamentarians and other community leaders, are not as knowledgeable about EC. Different brands of EC products are not known to KOLs, who stressed the need for informing communities about this product.

- Few KOLs are informed about ECP's mode of action ;

- Many KOLs indicated that EC is legal, available in the national program, and sold in private pharmacies ;

- Some KOLs cited low availability of EC products, particularly in public health facilities, which have experienced stock shortages since 2010 due to the expiration of the public sector ECP product, Pregnon ${ }^{\circledR}$;

- The vast majority of KOLs have also raised EC's high cost in private pharmacies, which remain first sources of supply for EC users ;

- Legislation should be revised similarly to other contraceptive products: EC products are among Table A products, therefore requiring a prescription from a doctor or a midwife ;

- Although KOLs do not have specific information on EC guidelines and protocols, most are in favor of integrating EC in the national guidelines for FP service provision;

- Few KOLs are aware of EC studies in Senegal and their findings. 
Attitudes, opinions and perceptions relating to EC use:

- Most KOLs think ECPs are useful, especially among young people, because they help prevent unwanted pregnancies after unprotected sex, also in cases of rapes and incest, as they can avoid health and social consequences such as induced abortion and infanticide ;

- KOLs indicated perceived reluctance among providers about offering EC services. A public official stated, "Among providers, there are still some stereotypes. They have not quite accepted this method. They are concerned about potential overuse. Providers are directing users towards other methods such as injections and implants ;"

- KOLs do not share uniform views of preferred users of EC. For some KOLs, "EC should be limited to certain women: those with high-riske pregnancies, women victims of rape and sexual assault, sex workers and women having casual sex (e.g. migrants' wives, teenagers)". Others indicated "EC should be available to all women who need it and as long as there is no medical contra-indication for the method;"

- KOLs urged caution in expanding EC access to the community level. A Ministry of Health official said, "For extension to the bealth posts, there are no problems. But for health buts (i.e. facilities lower than bealth posts), I suggest we wait for the results of the initial experience to be well established, because even health workers at this level may overuse it ;"

- KOLs believe repeat use of EC is a reality, seen especially in pharmacies. They also believe, however, that repeat use can also increase risk of STI exposure and substitute for regular contraception.

\section{Recommendations}

This study's primary recommendations include:

- Informing communities for wider product knowledge ;

- Training providers ;

- Establishing a logistics management system ensuring product availability ;

- Improving product affordability ;

- Developing effective partnerships, especially with pharmacists ;

- Increasing advocacy with decision makers. 


\section{Introduction}

Plus connue sous l'appellation de "pilule du lendemain", la Contraception d'Urgence (CU) est pratiquée depuis plus de 30 ans à travers le monde. La CU est une méthode contraceptive exceptionnelle qui permet d'éviter une grossesse non désirée après un rapport sexuel non ou mal protégé. C'est la seule méthode contraceptive utilisable immédiatement après qu'un rapport sexuel non protégé ait eu lieu. Parce qu'elle est très différente de toute autre méthode de l'éventail des produits contraceptifs, et parce qu'elle est efficace pour prévenir les grossesses chez les femmes qui l'utilisent, l'accès à la CU est une partie importante des stratégies globales de planification familiale, notamment dans un contexte où l'ampleur des grossesses non désirées, des violences et abus sexuels et leurs conséquences (avortements à risque, infanticides) interpelle de plus en plus l'attention voire la responsabilité des autorités publiques et de l'ensemble des acteurs de la société.

Malheureusement, il y a une méconnaissance généralisée sur ce produit, notamment sur le mécanisme d'action et l'effet de la CU sur la procréation. Ainsi, la diffusion, dans un format approprié, d'informations précises sur la $\mathrm{CU}$ requiert une haute priorité au niveau des programmes de santé de la reproduction en général, plus particulièrement des programmes de planification familiale (PF).

Au Sénégal, les initiatives de promotion de la CU demeurent très récentes. La première initiative a été parrainée par Equilibres et Population ${ }^{1}$, un partenaire du réseau ECafrique à travers une intervention pilote d'introduction de la CU dans les structures de l'ASBEF durant la période 2003-2005. Ce projet financé par la Fondation Compton ${ }^{3}$ fut conduit en partenariat avec HRA PHARMA ${ }^{4}$. L'objectif était d'introduire la $\mathrm{CU}$ comme moyen de prévention des grossesses non désirées, des avortements à risque et surtout comme tremplin pour les femmes vers l'utilisation d'une méthode régulière de contraception. Les activités ont porté sur le plaidoyer, l'IEC, la formation des prestataires et l'offre de services de CU. Ce projet pilote a permis de démontrer que l'utilité de la CU était bien perçue par le public et que les prestataires, une fois formés, trouvaient le produit facile à administrer. En 2005, ECafrique, en collaboration avec Equilibres et Population, lança l'initiative sous régionale sur la CU visant à consolider les leçons apprises de l'intervention pilote. Cette initiative sous régionale a contribué à engager le Ministère sénégalais de la santé et de la Prévention à mettre en œuvre les activités de formation, d'IEC/ plaidoyer en faveur de la CU. Avec l'appui de Population Council, plusieurs études ont été réalisées. La première étude réalisée en 2005, La CU: un aperç de la question au Sénégal, a permis de mettre en évidence la méconnaissance de la CU au niveau du public. La seconde étude, réalisée en 2006, est diagnostique qui a mis en évidence la nécessité de renforcer l'information sur la CU aussi bien au niveau des prestataires que de la communauté. En 2006, l'évaluation stratégique du programme national de PF révéla que les prestataires aussi bien que les clientes avaient une connaissance limitée sur la CU. Cette évaluation avait par ailleurs montré que l'offre de service de CU était limitée à quelques centres de santé et que la grande majorité des prestataires n'avait aucune formation spécifique sur la CU. Ces résultats ont été corroborés par l'Etude d'Analyse Situationnelle (AS) des services de santé de la reproduction (SR), réalisée en 2007, qui avait montré que seul 10\% des Points de Prestations de Services (PPS) disposaient de produits spécifiques à la CU et que 37\% des prestataires interviewés n'avaient pas reçu de formation en CU.

En 2008, en collaboration avec le CEFOREP, une étude sur la prévention des grossesses non désirées en milieu scolaire dans la zone de Pikine-Guédiawaye a permis d'identifier la formation des prestataires, la sensibilisation des adolescents, l'amélioration de la disponibilité et de l'accès des produits CU comme actions clés à entreprendre dans la perspective de la promotion de la CU.

Les résultats de ces recherches ont contribué à faciliter l'intégration de la $\mathrm{CU}$ dans les politiques et normes de services de santé de la reproduction. Déjà en 1998, la CU a été introduite dans le curriculum de formation des prestataires de services. En 2000, la CU fut mentionnée pour la première fois dans les Politiques, Normes et Protocoles (PNP). Ces PNP mentionnaient que les Contraceptifs Oraux Combinés, les Pilules progestatives et le DIU pouvaient être utilisées comme méthodes dans le cadre d'une CU. En 2005, le Pregnon fut introduit comme la première méthode spécifique de CU dans les PNP.

\footnotetext{
1 Equilibres et Population est une ONG basée à Paris et qui a été créée en 1993 par des médecins et des journalistes en vue de promouvoir le bon usage de l'aide publique au développement 2 Le Forum africain sur la Contraception d'Urgence ou ECafrique est un réseau de professionnels intervenant dans le domaine de la santé qui vise à accroitre la disponibilité des services de CU de qualité en Afrique. Ce réseau crée en 2002 est sponsorisé par Population Council une ONG américaine basée à New York.

3 La fondation Compton est une organisation à but non lucratif basée aux Etats Unis 5 Californie) qui vise à assurer la sécurité bumaine et environnementale et à préserver les droits des générations futures.

4 HRA PHARMA est une institution qui a pour objectif de développer et commercialiser des médicaments ou dispositifs médicaux répondant à de réels besoins en matière de santé mais ne soulevant pas l'intérêt des groupes pharmaceutiques. Elle a développé le Norlevo qui est un produit de CU.
} 
A travers l'appui de Population Council, le Ministère de la santé a pu ainsi former durant la période 2006-2010, près de 1200 prestataires, pharmaciens et assistants pharmaciens et relais communautaires sur l'offre de CU et l'éducation des clients à l'utilisation de ce produit. Des supports didactiques ont été développés et distribués aux prestataires et relais communautaires dans plusieurs régions. L'introduction du Pregnon avec l'appui de l'UNFPA dans les PPS du secteur public a permis de renforcer l'accessibilité de la CU dans le système national de santé. Au niveau du secteur privé, la PRODIPHARMA a assuré depuis 2005 la distribution et la promotion du Norlevo.

En 2009, l'étude réalisée auprès des pharmacies privées a révélé que les officines privées demeuraient la principale source d'approvisionnement en produits de $\mathrm{CU}$ et mis en évidence la nécessité d'harmoniser les procédures d'offre de $\mathrm{CU}$ au niveau du secteur privé. Cette étude a montré par ailleurs le besoin d'assurer une mise à jour des pharmaciens et assistants pharmaciens sur les nouveaux protocoles d'offre de CU.

En 2010, la Division de la santé de la Reproduction, avec l'appui de Population Council, a procédé à l'évaluation du processus d'intégration de la CU dans le système national de santé, dans le cadre du forum national sur la CU. Ce forum avait révélé que la CU souffrait d'un problème réel de promotion dans le système national de santé, ceci malgré les efforts consentis.

En effet, il a été noté:

- Une méconnaissance généralisée du produit au niveau des populations ;

- La persistance des rumeurs autour de la CU qui s'explique par la spécificité et par la sensibilité du produit ;

- Une faible prise en compte de la CU dans l'offre de service de routine, notamment lors du counseling initial et dans les campagnes d'IEC/PF ;

- Bien que les officines privées soient la principale source d'approvisionnement des utilisateurs de CU, les prestataires de services au niveau des pharmacies privées avaient besoin d'une mise à jour sur les nouveaux protocoles d'offre de CU.

L'utilisation de la $\mathrm{CU}$ au niveau du système public demeure encore très faible si l'on se réfère aux statistiques de routine $(<2 \%)$. Au niveau du secteur privé, l'absence d'un système d'information fonctionnel ne permet pas d'apprécier l'utilisation réelle de la CU au niveau des pharmacies.

L'offre de services de CU dans le secteur public continue d'être entravée par une insuffisance dans la promotion de ce produit. Les croyances, les attitudes et pratiques des populations en général, des prestataires en particulier freineraient la promotion et l'utilisation de ce produit chez les groupes qui en ont surtout besoin.

Ainsi, dans la perspective de repositionner la CU dans les programmes de PF, Population Council a mené, en collaboration avec la Division de la Santé de la Reproduction (DSR), une étude sur les attitudes, croyances et pratiques des leaders d'opinion et des prestataires autour de la CU.

Cette étude entre dans le cadre d'une étude multicentrique incluant le Nigéria, l'Inde et le Sénégal. Ces trois pays bénéficient d'un financement de la Fondation Bill \& Melinda Gates en soutien à la mise en œuvre de l'Initiative de Santé Urbaine en cours dans ces pays.

\section{But de l'Etude}

Proposer des stratégies novatrices pour repositionner la CU dans les secteurs de santé public et privé.

\section{Objectifs de l'etude}

\section{Objectif général}

L'objectif global de l'étude est de recueillir des informations auprès des leaders d'opinion et des prestataires sur leurs connaissances, attitudes et pratiques concernant la $\mathrm{CU}$, et de saisir leurs options et perceptions qui peuvent avoir une influence sur les questions politiques et programmatiques liées à la CU. 


\section{Objectifs spécifiques}

- Documenter les attitudes, croyances et pratiques des prestataires autour de la CU, notamment sur la question de l'utilisation répétée ;

- Capturer les opinions et perceptions des leaders d'opinion clés sur les questions de politiques et programmes relatifs à la $\mathrm{CU}$;

- Recueillir leurs suggestions et recommandations en vue d'asseoir des interventions pertinentes pour le repositionnement de la CU dans les secteurs de santé public et privé.

\section{Méthodologie}

\section{Sites de l'étude}

- L'étude s'est déroulée durant la période d'août-septembre 2011 dans les centres urbains des régions de Dakar et de Thiès qui sont des zones ciblées par le projet "Initiative Sénégalaise de Santé Urbaine" (ISSU) financé par la Fondation Bill et Melinda Gates. Le choix de ces sites offre une bonne opportunité dans la synergie des interventions avec le projet ISSU.

Le choix des villes de Dakar, Thiès et Mbour se justifie par:

- Dakar: le choix de Dakar se justifie par son poids important dans la dynamique démographique et urbaine au Sénégal et par sa représentativité des problèmes sociaux et économiques du pays ;

- Thiès: le choix de la ville de Thiès se justifie par son poids démographique en tant second centre urbain au niveau national et par le besoin d'avoir une situation de l'offre de CU en dehors de Dakar. La ville de Thiès abrite par ailleurs un hôpital régional et plusieurs cliniques privées ;

- Mbour: a été inclus en tant que centre urbain émergeant avec un profil économique et social différent des deux autres villes. Mbour se caractérise par son profil touristique qui expose les adolescents/jeunes aux problèmes cruciaux liées à la sexualité précoce et ses conséquences sur les grossesses non désirées.

\section{Cible de l'étude}

L'étude a ciblé deux types de populations:

- Les Leaders d'Opinion Clés $\left[\mathrm{LOC}^{5}\right.$ : cette cible comprend les autorités publiques (représentants des Ministères de la Santé, de l'Education Nationale, de la Jeunesse, du Genre...), les membres du parlement, les membres d'associations professionnelles (ordre des Pharmaciens, des Médecins, association des sages-femmes, femmes juristes), les représentants des partenaires au développement, les membres d'ONGs locales, les réseaux professionnels et religieux (Réseau des Journalistes, Islam et Population et Réseau Siggil Jiggen), les distributeurs, les membres des Organisations Communautaires de Base [OCB], notamment les associations de femmes, de jeunes, et les praticiens du secteur privé. Le choix de ces LOC se justifie par le fait que ces derniers jouent un rôle clé dans la définition des stratégies et des politiques nationales et dans l'allocation des ressources ; ils bénéficient d'une grande écoute auprès des populations et peuvent influencer les opinions. Leurs décisions comptent, d'où l'intérêt d'avoir leurs opinions et suggestions en vue de bâtir un plaidoyer solide dans la perspective du repositionnement de la CU;

- Lesprestataires de services : l'enquête auprès des prestataires a ciblé une gamme variée de prestataires intervenant dans l'offre de services de PF tant au niveau du secteur public, privé et parapublic. Il s'agit notamment de médecins, de sages-femmes, d'infirmiers/assistants infirmiers, de pharmaciens et agents de comptoir, des prestataires intervenant au niveau des centres conseils adolescents, des cliniques privées, des cliniques d'Ongs, des infirmeries des écoles.

\section{Sélection des cibles}

Les leaders d'opinion clés : il a été procédé à un choix raisonné des LOC; ce choix a été basé sur l'intérêt et l'implication de leurs institutions d'appartenance par rapport à la CU et sur le rôle et l'influence que ces institutions pouvaient jouer dans le repositionnement de la CU. 
Les prestataires : au premier degré, on a procédé à la sélection des (PPS offrant habituellement les services de PF y compris la CU. Le choix des PPS a tenu compte du niveau de représentativité des types de PPS (public/privé/parapublic) et du niveau de fréquentation des PPS par les populations. Ces PPS comprennent les structures sanitaires du secteur public (hôpitaux, centres et postes de santé), du secteur parapublic (Centre Social du COUD, Dakar Dem Dikk, Caisse de Sécurité Social) les cliniques et pharmacies privées, les cliniques des ONG (de l'ASBEF, de l'ACDEV), les Centres Conseils Adolescents. Au second degré, les prestataires ayant déjà eu à offrir une méthode de CU dans leur passé professionnelle et ceux qui continuent encore de l'offrir ont été inclus dans l'étude et interviewés après avoir sollicité leur accord préalable. Au moins un prestataire par PPS a été interviewé.

\section{Instruments de collecte et questions explorées}

Leaders d'opinion clés: un guide d'entretien semi structuré a été administré aux LOC ; les questions explorées ont porté sur la connaissance du produit (son statut juridique, son mode d'action, son efficacité, le délai d'utilisation), l'offre de service de CU, la CU dans les politiques, normes et protocoles, la recherche en CU, les perceptions, attitudes et pratiques sur le produit, les obstacles liés à l'offre et à l'accès, l'utilisation de la CU, les suggestions pour la promotion de la CU. Les interviews ont été conduites par le coordonnateur de l'étude avec l'appui d'un sociologue consultant.

Les prestataires : un questionnaire individuel quantitatif a été administré aux prestataires. Comme pour les LOC, les questions ont porté sur la connaissance du produit, leurs perceptions, attitudes et pratiques par rapport à la CU, à l'utilisation de la CU, leur expérience par rapport à l'offre de CU. Les interviews ont été conduites par des coordinatrices SR qui ont été formées au préalable à l'administration des questionnaires.

\section{Considérations éthiques}

Aussi bien pour les LOC que pour les prestataires, un formulaire de consentement éclairé a été soumis avant le début de l'interview. La participation à l'enquête a été volontaire et aucune rémunération n'a été donnée aux participants. Les interviews se sont déroulées dans la confidentialité et l'anonymat des personnes interviewées a été préservé pendant l'enquête et au moment de l'exploitation des données en affectant des codes à toutes les informations personnelles liées aux participants. Aucun nom n’a été enregistré.

\section{Gestion et analyse des données}

L'enquête auprès des prestataires : la saisie des données a été faite avec le logiciel Epidata; ces données ont été transférées sous SPSS pour les besoins de l'analyse des données. Des analyses univariées et bivariées ont été faites.

L'enquête auprès des LOC: l'analyse de contenu a été utilisée pour l'exploitation des informations qualitatives recueillies auprès des LOC. Cette analyse a été faite autour des thèmes similaires pour permettre une comparabilité entre les trois pays concernés par l'étude.

\section{Bilan de la Collecte}

\section{Les Leaders d'Opinion Clés (LOC)}

Le tableau un indique la répartition des LOC selon le profil. Au total 34 LOC ont été interviewés dont 11 intervenant au niveau d'institutions publiques (responsables des Ministères de la Santé, de l'Education, de la Justice, de la jeunesse, membres du parlement...), cinq membres d'associations professionnelles ou de religieux (association des femmes juristes, association des sages-femmes, réseau Islam et Population...), les représentants des partenaires au développement (4), les représentants des institutions impliquées dans la distribution des produits de la $\mathrm{CU}$ (Pharmacie Nationale d'Approvisionnement, responsable logistique de la DSR, PRODIPHARM), les membres d'Organisations Communautaires de Base (OCB) tels que Synergie banlieue, Mouvement d'Action pour les Jeunes (MAJ) et les praticiens privés. Le choix varié des différentes institutions ciblées dans cette étude répondait au besoin d'avoir une image diversifiée d'opinions des principaux responsables d'institutions (décideurs publiques, société civile, communauté, ONG...) qui pourraient jouer un rôle déterminant dans les choix politiques et programmatiques en vue du repositionnement de la CU. 
Tableau 1: Répartition des LOC selon le profil

\begin{tabular}{|l|c|}
\hline Profil des LOC & Nombre \\
\hline Décideurs au niveau politique/programmatique & 11 \\
\hline Membres associations professionnelles & 5 \\
\hline Membres d'ONGs & 3 \\
\hline Partenaires au développement & 4 \\
\hline Praticiens & 1 \\
\hline Distributeurs & 3 \\
\hline Membres d'Organisations Communautaires de Base (OCB) & 7 \\
\hline Total & $\mathbf{3 4}$ \\
\hline
\end{tabular}

Source: enquête auprès des leaders d'opinion Clés, juillet 2011

\section{Les prestataires}

L'enquête auprès des prestataires a couvert une gamme variée de PPS comprenant des structures sanitaires du secteur public, privé et parapublic. Cent neuf PPS ont été visités dont 36 pharmacies privées, 26 postes de santé, 13 cliniques privées, 15 centres de santé. L'échantillon inclut également des structures spécifiques telles que les centres conseils pour adolescents (4), les infirmeries des écoles qui sont des structures destinées spécifiquement aux adolescents/jeunes en milieu extrascolaire et scolaire (2). L'essentiel des PPS est concentré dans la région de Dakar (82\% des PPS).

Tableau 2: Répartition des Points de Prestations de services selon le type

\begin{tabular}{|l|c|c|c|c|}
\hline Type de structures visitées & Dakar & Thiès & Mbour & Ensemble \\
\hline Hôpital & 4 & 1 & & 5 \\
\hline Centre de référence PF & 1 & & & 1 \\
\hline Centre de santé & 14 & & 1 & 15 \\
\hline Poste de santé & 20 & 4 & 2 & 26 \\
\hline Centre Conseils Ado & 3 & & 1 & 4 \\
\hline Clinique privée & 10 & 2 & 1 & 13 \\
\hline Clinique ONG & 2 & & & 2 \\
\hline Pharmacie/ Officine privée & 30 & 4 & 2 & 36 \\
\hline EPS 1 & 3 & 1 & 1 & 5 \\
\hline CMS & 2 & & & 2 \\
\hline Total & $\mathbf{8 9}$ & $\mathbf{1 2}$ & $\mathbf{8}$ & $\mathbf{1 0 9}$ \\
\hline
\end{tabular}

Source: enquête auprès des prestataires, septembre 2011

Au niveau de ces 109 PPS visités, 155 prestataires ont pu être interviewés. Les sages-femmes représentent la majorité de l'échantillon (54\%); la forte présence des sages-femmes dans l'échantillon s'explique par le fait que ce sont les prestataires de première ligne en ce qui concerne l'offre de services de PF ; les agents de comptoir au niveau des pharmacies constituent 19\% de l'échantillon suivis des pharmaciens (17\%) et des médecins $(6 \%)$. Les autres catégories sont faiblement représentées dans l'échantillon.

Tableau 3: Répartition des prestataires selon la catégorie professionnelle

\begin{tabular}{|l|c|c|c|c|}
\hline Type de prestataires & Dakar & Thiès & Mbour & Ensemble \\
\hline Sage-femme & 68 & 10 & 5 & $\mathbf{8 3}$ \\
\hline Pharmacien & 21 & 3 & 2 & $\mathbf{2 6}$ \\
\hline Médecin & 8 & 1 & 1 & $\mathbf{1 0}$ \\
\hline Agent de comptoir & 28 & 2 & & 30 \\
\hline Autre & 4 & & 2 & $\mathbf{6}$ \\
\hline Total & $\mathbf{1 2 9}$ & $\mathbf{1 6}$ & $\mathbf{1 0}$ & $\mathbf{1 5 5}$ \\
\hline
\end{tabular}

Les femmes constituent l'essentiel de l'échantillon des prestataires $(71,6 \%)$. On observe à Mbour que la part des hommes est plus importante (40\%) comparée à Thiès et Dakar (27,9\% et $25 \%$ respectivement).

Tableau 4: Répartition des prestataires selon le sexe

\begin{tabular}{|l|c|c|c|c|c|c|c|c|}
\hline \multirow{2}{*}{ Sexe } & \multicolumn{2}{|c|}{ Dakar } & \multicolumn{2}{c|}{ Thiès } & \multicolumn{2}{c|}{ Mbour } & \multicolumn{2}{c|}{ Ensemble } \\
\cline { 2 - 9 } & $\mathbf{N}$ & $\mathbf{\%}$ & $\mathbf{N}$ & $\mathbf{0}$ & $\mathbf{N}$ & $\mathbf{\%}$ & $\mathbf{N}$ & $\mathbf{\%}$ \\
\hline Masculin & 36 & 27.9 & 4 & 25 & 4 & 40 & 44 & 28,4 \\
\hline Féminin & 93 & 72.1 & 12 & 75 & 6 & 60 & 111 & 71,6 \\
\hline Total & $\mathbf{1 2 9}$ & $\mathbf{1 0 0}$ & $\mathbf{1 6}$ & $\mathbf{1 0 0}$ & $\mathbf{1 0}$ & $\mathbf{1 0 0}$ & $\mathbf{1 5 5}$ & $\mathbf{1 0 0}$ \\
\hline
\end{tabular}

L'âge moyen des prestataires est de 43,50 ans avec un âge minimum de 25 ans et un âge maximum de 70 ans. Le nombre moyen de mois d'expérience en matière d'offre de CU est de 59.4 mois, soit près de cinq ans d'expérience, ce qui correspond à la période de démarrage des activités de formation des prestataires dans le cadre du programme national de PF qui ont débuté en 2005. Toutefois, le tableau cinq indique que certains prestataires ont une expérience largement antérieure (22 ans) à l'initiative d'introduction de la CU dans le système national de santé qui remonte à moins de 10 ans avec l'introduction du Pregnon et 
du Norlevo respectivement dans les secteurs public et privé. Ces prestataires utilisaient certainement les pilules classiques et peut être le dispositif intra-utérin (DIU) qui étaient les méthodes dédiées auparavant pour la CU.

Tableau 5: Répartition des prestataires selon l'âge et l'expérience en matière de CU

\begin{tabular}{|c|c|c|c|c|}
\hline & Dakar & Thiès & Mbour & Ensemble \\
\hline \multicolumn{5}{|c|}{ Age des prestataires (années) } \\
\hline $\mathrm{N}$ & 129 & 16 & 10 & 155 \\
\hline Moyenne & 44 & 43 & 44 & 44 \\
\hline Minimum & 25 & 29 & 31 & 25 \\
\hline Maximum & 70 & 59 & 56 & 70 \\
\hline \multicolumn{5}{|c|}{ Nombre de mois d'expérience en matière de CU } \\
\hline $\mathrm{N}$ & 111 & 15 & 10 & 136 \\
\hline Moyenne & 58 & 45 & 102 & 59 \\
\hline Minimum & 0 & 1 & 36 & 0 \\
\hline Maximum & 264 & 120 & 150 & 264 \\
\hline
\end{tabular}

Le tableau six indique une quasi-effectivité de l'offre de service de CU. Parmi les 155 prestataires qui ont eu à offrir la CU dans le passé, $92 \%$ continuent d'offrir la CU. Toutefois, on note que 11 sages-femmes sur les 83 ayant déjà offert la CU ne l'offrent plus.

Tableau 6: Pourcentage de prestataires offrant actuellement la CU selon la catégorie professionnelle et la région

\begin{tabular}{|l|c|c|c|c|c|c|c|c|}
\hline \multirow{2}{*}{ Catégorie professionnelle } & \multicolumn{2}{|c|}{ Dakar } & \multicolumn{2}{|c|}{ Thiès } & \multicolumn{2}{c|}{ Mbour } & \multicolumn{2}{c|}{ Ensemble } \\
\cline { 2 - 9 } & $\mathbf{N}$ & $\mathbf{0}$ & $\mathbf{N}$ & $\mathbf{0}$ & $\mathbf{N}$ & $\mathbf{\%}$ & $\mathbf{N}$ & $\mathbf{\%}$ \\
\hline Sage-femme & 58 & 85,3 & 9 & 90,0 & 5 & 100 & 72 & 86,7 \\
\hline Pharmacien & 21 & 100 & 3 & 100 & 2 & 100 & 26 & 100 \\
\hline Médecin & 8 & 100 & 1 & 100 & 1 & 100 & 10 & 100 \\
\hline Agent de comptoir & 27 & 96,4 & 2 & 100 & & & 29 & 96,7 \\
\hline Autre & $\mathbf{4}$ & $\mathbf{1 0 0}$ & & & $\mathbf{2}$ & $\mathbf{1 0 0}$ & $\mathbf{6}$ & $\mathbf{1 0 0}$ \\
\hline Total & $\mathbf{1 1 8}$ & $\mathbf{9 1 , 5}$ & $\mathbf{1 5}$ & $\mathbf{9 3 , 8}$ & $\mathbf{1 0}$ & $\mathbf{1 0 0}$ & $\mathbf{1 4 3}$ & $\mathbf{9 2 , 3}$ \\
\hline
\end{tabular}

\section{Les Leaders d'Opinion Cles (LOC): Qui Sont-ils?}

Le choix des LOC a été motivé par le rôle majeur que ces derniers jouent dans la construction des idées et des opinions au sein de la société et surtout par leur rôle dans les prises de décision au niveau social, politique et économique. Les LOC jouent en effet un rôle clé dans la définition des politiques et stratégies nationales. Ce sont eux qui fixent les priorités nationales, notamment dans l'allocation des ressources. Leurs décisions comptent, d'où l'importance de mieux saisir leurs opinions et perceptions sur des sujets d'intérêt social majeur tels que la planification familiale et la contraception d'urgence. L'intérêt d'impliquer les LOC dans les stratégies de repositionnement de la CU se justifierait par le fait qu'ils peuvent être soit des partenaires clés ou des pourfendeurs de la CU. L'intégration des LOC dans les programmes demeure un enjeu fondamental pour le plaidoyer en faveur de la CU.

Dans le cadre de cette étude, une gamme variée de LOC a été ciblée allant des décideurs/gestionnaires de programmes au niveau politique, des membres des associations professionnelles, des réseaux professionnelles, des membres d'ONG locales, des partenaires au développement, des représentants des OCB, des distributeurs, des praticiens privés.

Tableau 7: Liste des institutions visitées

\begin{tabular}{|c|c|}
\hline INSTITUTIONS & INSTITUTIONS \\
\hline $\begin{array}{l}\text { Décideurs publiques : } \\
\text { - Ministère de la Santé et de la Prévention (DSR) } \\
\text { - Ministère de l'Education Nationale } \\
\text { - Ministère de la jeunesse } \\
\text { - L'Assemblée Nationale }\end{array}$ & $\begin{array}{l}\text { Les ONGs locales : } \\
\text { • ASBEF } \\
\text { • Action pour le Développement (ACDEV) }\end{array}$ \\
\hline $\begin{array}{cl}\text { Les partenaires au développement : } \\
\text { • } & \text { OMS } \\
\text { • } & \text { UNFPA } \\
\text { • } & \text { Marie Stopes International (MSI) }\end{array}$ & $\begin{array}{l}\text { Les praticiens : } \\
\qquad \quad \text { Un médecin privé }\end{array}$ \\
\hline $\begin{array}{l}\text { Les associations de professionnelles: } \\
\qquad \quad \text { Ordre des médecins du Sénégal } \\
\cdot \quad \text { Ordre des pharmaciens du Sénégal } \\
\cdot \quad \text { Association Nationale des sages-femmes du Sénégal }\end{array}$ & $\begin{array}{l}\text { Les OCB : } \\
\text { • Le réseau Siggil Jiggen } \\
\text { • La FAWE } \\
\text { • Le Mouvement d'Action pour les Jeunes } \\
\quad \text { Synergie Banlieue }\end{array}$ \\
\hline
\end{tabular}




\begin{tabular}{|c|c|}
\hline INSTITUTIONS & INSTITUTIONS \\
\hline \multirow[t]{2}{*}{$\begin{array}{l}\text { Les réseaux : } \\
\qquad \quad \text { Réseau Islam et Population (religieux) } \\
\cdot \quad \text { Réseau des journalistes en santé et Population }\end{array}$} & 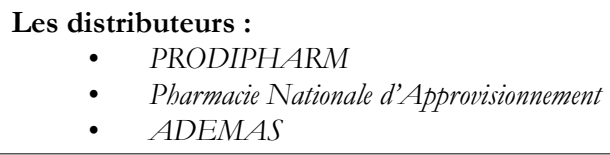 \\
\hline & $\begin{array}{l}\text { La police : } \\
\qquad \quad \text { Prison des femmes du Camp pénal1 }\end{array}$ \\
\hline
\end{tabular}

\section{Connaissance du Produit}

\section{Informations générales sur le produit}

En dehors des LOC qui sont directement impliqués dans la gestion de routine des programmes de santé de la reproduction, notamment les leaders au niveau du Ministère de la Santé, du Ministère de l'Education nationale, les partenaires au développement, les membres des ONG locales, la plupart des LOC n'ont pas une information précise sur la CU. Ils la connaissent plutôt sous le nom de "pilule du lendemain" mais ils ne peuvent ni citer les différentes marques de produits de CU disponibles dans le pays, ni parler de son mode d'action, de ses effets secondaires. C'est le cas chez les parlementaires, les réseaux des journalistes, les religieux, et certains membres d'OCB. Il importe donc d'accroître la connaissance de ces LOC sur la CU en leur donnant des informations plus spécifiques sur le produit. Par ailleurs, il importe de renforcer la sensibilisation de ces leaders sur l'utilité et l'innocuité du produit. Ceci pourrait ainsi contribuer à l'adhésion de ces leaders à la politique de promotion de la CU.

\section{Les produits de CU les plus connus}

Selon les LOC, les principaux produits de CU disponibles sont les suivants:

- Le Norlevo ${ }^{\circledR}(1,5 \mathrm{mg})$ qui a été mis à la disposition de la DSR avant 2006, mais actuellement ce produit n'est disponible qu'au niveau des officines privées ;

- Le Pregnon ${ }^{\circledR}$ qui a été introduit dans le système public par le programme national de PF depuis le 16/05/2006 grâce à une dotation de l'UNFPA. Cependant ce stock disponible est périmé depuis septembre 2010 faute de promotion et de distribution par les prestataires du secteur public ;

- Le DUET qui est le nouveau produit qui va remplacer le Pregnon ${ }^{\circledR}$;

- L'Optinor qui n'est disponible qu'au niveau des structures de l'ASBEF;

- Les pilules classiques (Loféménal, Microval, ovrette...);

- Le DIU.

\section{Statut juridique de la CU}

La grande majorité des LOC pense que les produits de la CU sont légaux du fait qu'ils sont disponibles au niveau du programme national de planification familiale au même titre que les autres produits contraceptifs et qu'ils sont vendus dans les pharmacies privées. Ainsi parlant du Norlevo ${ }^{\circledR}$, un membre d'associations professionnelles indiquait que "C'est un produit autorisé ... Comme pour les autres produits que l'on retrouve dans les officines, il a eu un visa de la Direction des Pharmacies et Laboratoires" [membre d'association professionnelle]. Toutefois, selon certains LOC, bien que les produits de la CU soient autorisés, il convient de revoir la réglementation pharmaceutique relative aux contraceptifs en général et à la $\mathrm{CU}$ en particulier. Un membre d'une association professionnelle déclarait que "Le Norlevo $0^{\circledR}$ fait partie de la liste des médicaments du tableau $A$ et doit être vendue sous ordonnance mais que dans la réalité le produit se livre à la demande" [un membre du réseau des distributeurs]. Selon ces LOC, cette situation devrait être corrigée car actuellement, "Selon les dispositions réglementaires, seuls les médecins et sages-femmes sont autorisés à prescrire cette méthode; cependant, dans la pratique, il a été noté que des infirmiers délivrent le produit ... il importe donc de lever certaines de ces contradictions" [un distributeur]. 


\section{Utilité du produit}

Tous les LOC sont unanimes sur l'utilité du produit et pensent que le produit doit être vulgarisé pour plusieurs raisons:

- "Pour que tout le monde ait l'information ;"

- "C'est un produit efficace pour prévenir une grossesse non désirée ainsi que des cas de décès et certaines situations sociales (infanticides, abandons, avortements clandestins et leurs conséquences sociales) ;"

- "C'est un produit qui est utile et qui a sa place dans le système pour capturer les besoins non satisfaits ;"

- "Elle peut aider les jeunes à mieux gérer leur avenir ;"

- "Facile à utiliser ;"

- "Il y a des femmes qui, pour différentes raisons, n'utilisent pas les méthodes régulières."

Certains LOC justifient l'utilité du produit par le fait “qu'il peut aider à lutter contre les taux élevés d'abandon des filles à l'école et par le fait qu'elle permet d'aider certaines organisations de lutte contre les violences sexuelles à mieux prendre en charge les victimes de ces abus/violences sexuelles" [un membre d'association professionnelle].

Bien qu'ils soient favorables à la vulgarisation de ce produit, certains LOC laissent apparaitre leurs craintes quant et pensent que son utilisation devrait être encadrée pour "qu'elle ne soit utilisée comme la seule et bonne méthode" [un décideur].

Certaines réserves chez les LOC s'expliqueraient par des raisons personnelles: "Personnellement je pense que son utilisation doit être circonscrite pour plusieurs raisons : en tant que musulmane, mère de famille et éducatrice. Les valeurs socioculturelles ne peuvent pas être bafouées d'un seul coup. Avec cette méthode, il faut faire attention à ne pas heurter certaines consciences. De ce fait, il faut impliquer les religieux. Mais dans des situations critiques (viols, incestes) on peut l'utiliser pour aider certains jeunes, ne serait-ce que sur le plan psychologique" [un décideur].

\section{Efficacité et innocuité du produit}

Selon les LOC, la CU est jugée efficace et sûre si elle est bien prise et au moment opportun. Et également si les conditions de prise et de conservation sont respectées. Et à priori tous les prestataires maitrisent la CU dans la mesure où ils ont été formés. Les LOC justifient également l'efficacité du produit par la forte demande au niveau des pharmacies privées et aussi par le fait que peu de cas d'échec et de plaintes ont été évoqués. Seul, un membre d'OCB a évoqué un cas d'échec. Concernant les effets secondaires, les LOC ne les excluent pas mais pensent qu'ils sont mineurs. Selon certains LOC, "Cette innocuité du produit est réelle car avant son introduction, comme pour tout médicament, il a regu un avis de la DPL" [un membre d'association professionnelle].

Les LOC n'excluent pas la probabilité de l'existence de produits de CU de contrefaçon dans un contexte où le marché, notamment dans le secteur informel, est très poreux du fait de l'inexistence d'un système de contrôle efficace. Selon un LOC, "Comme tout médicament, les produits de la CU peuvent faire l'objet de contrefaçon" [Un décideur]. Aucun LOC n’a signalé avoir vu ou eu écho de l'existence d'un produit de contrefaçon. Ces LOC se fient surtout aux résultats des contrôles effectués: "Tous les produits contraceptifs ontété enrôlés dans le contrôle qualité et qu'aucun produit contrefait n'a été signalê" [un gestionnaire de programme].

\section{La CU dans les Politiques/normes et directives nationales}

La CU a été intégrée dans les derniers documents de Politiques/Normes et Protocoles (PNP) en matière de Planification Familiale/Santé de la Reproduction révisés en 2007-2008. Elle figure par ailleurs en bonne place dans la nouvelle version des PNP qui sera diffusée en 2012. Il faut noter que la plupart des LOC sont favorables à l'intégration de la $\mathrm{CU}$ dans les directives nationales dans la mesure où "la $C U$ fait partie de la gamme des produits contraceptifs disponibles dans le programme national de PF" [un manageur de programme/décideur]. Toutefois, les LOC sont peu informés de l'existence de ces directives nationales.

\section{Source d'approvisionnement/système logistique}

L'approvisionnement du pays en produits CU se fait à travers deux systèmes. Au niveau du secteur public, l'approvisionnement suit le système officiel des produits contraceptifs et se fait par la DSR à travers un soutien des partenaires au développement, principalement de l'UNFPA qui depuis 2006 appuie la DSR pour la mise en place des produits (le Pregnon $\left.{ }^{\circledR}\right)$. Dans une première étape, la DSR acheminait directement les produits au niveau des régions médicales. Les districts, qui constituent 
le niveau opérationnel de mise en œuvre des activités, s'approvisionnent au niveau de leurs régions médicales. Depuis 2010, à travers une convention signée entre la DSR et la Pharmacie Nationale d'Approvisionnement (PNA), cette dernière achète pour la DSR et assure l'approvisionnement et la distribution selon les recommandations de la division y compris les produits en donation. Un système de tarification des prix des produits contraceptifs y compris de la CU a été validé par le Ministère de la Santé qui a fixé et publié les prix officiels de cession des produits contraceptifs à tous les niveaux du système de santé. L'absence de transmission des données de consommation des produits contraceptifs à travers les Registres Trimestriels de Stocks (RTS) constitue un maillon faible du système d'approvisionnement dans la mesure où le niveau de consommation réelle n'est pas connu, ce qui limite la qualité des commandes de produits. Depuis 2010, le système a connu une rupture des produits due à la péremption du Pregnon ${ }^{6 \circledast}$ du fait de l'absence de promotion par les prestataires. Actuellement, un nouveau produit dénommé DUET a remplacé le Pregnon ${ }^{\circledR}$ dans le système public. La dotation de DUET a été commandée par la PNA dans le cadre de la convention mais les quantités disponibles ne permettent pas de couvrir les besoins annuels du pays. L'offre de CU est assurée par un personnel qualifié au niveau de l'ensemble des structures publiques sauf au niveau des cases de santé. Généralement, ce sont surtout les gynécologues et les sages-femmes qui offrent ce produit.

Au niveau du secteur privé, la CU est vendue au niveau des pharmacies privées sous la marque Norlevo ${ }^{\circledR}$. Au niveau des cliniques privées, ce sont les médecins et sages-femmes qui offrent généralement la CU sur la base d'une prescription aux clients qui vont chercher le produit au niveau des pharmacies privées. Le Norlevo ${ }^{\circledR}$ est rétrocédé aux clients à 3275 FCFA. Cinq principaux grossistes se partagent le marché privé: PRODIPHARM, LABOREX, SODIPHARM, COPHASE, BIOPHRAM. Selon les estimations, "près de 80000 unités de Norlevo ${ }^{\circledR}$ sont consommées chaque année au niveau des pharmacies privées ... la région de Dakar représente près de 60\% du marché national ... Le produit est surtout utilisé en ville" [un distributeur].

Au niveau de l'ASBEF, “c'est l'Optinor ${ }^{\circledR}$ qui est disponible grâce à l'appui de l'IPPF. Il est vendu à 2000 FCFA" [un membre d'ONG].

\section{La recherche sur la CU}

Peu de LOC sont informés de l'existence des études faites au Sénégal et de leurs résultats. Les principales études qui ont été citées par les LOC avertis sont:

- La CU: Un aperçu de la question au Sénégal (Population Council 2005) ;

- Etude diagnostique sur la CU (CEFOREP/2006);

- Etude sur la prévention des grossesses non désirées en milieu scolaire dans la zone de Pikine-Guédiawaye (Population Council et CEFOREP 2007) ;

- Enquête sur la CU au niveau des pharmacies privées (Population Council 2009).

Certains LOC ont exprimé des pistes pour couvrir certaines préoccupations de recherche:

- Acceptabilité de la CU au niveau des populations, des décideurs ;

- Stigmatisation des utilisateurs de la CU;

- Aspects religieux liés au recours de la CU;

- Efficacité et innocuité du produit ;

- Perception des parents, de la communauté quant à l'utilisation de la CU par les adolescents/ jeunes non mariées ;

- Effets de l'usage répété/abusif ;

- Utilisation de la CU et exposition au VIH/SIDA ;

- Enquête CAP auprès des jeunes ;

- Enquête CAP auprès des prestataires.

6 Près de 25000 unités de Pregnon sont entrées en péremption du fait de la non distribution du produit par les prestataires. Cette rupture est le reflet des dysfonctionnements constatés en 2011 au niveau du système global d'approvisionnement et de gestion des produits contraceptifs. 


\section{Attitudes, Opinions et Perceptions des LOC sur la CU}

\section{Acceptabilité du produit}

Globalement les LOC ont une bonne opinion de la CU. Ils pensent que cette méthode a sa place dans la gamme des contraceptifs et peut constituer un recours dans certaines circonstances (oublis de pilule, viols, incestes...). Selon certains, "Elle devrait être promue même chę les jeunes célibataires car elle permet de prévenir les grossesses non désirées (surtout en cas d'oubli, de viol, d'inceste) et de leurs conséquences sociales (avortements provoqués, infanticides)" [*** auteur].

Ceci est confirmé par les propos de certains LOC:

"C'est une méthode dont on a besoin dans le cadre de nos activités car les grossesses non désirées peuvent avoir des conséquences sociales très graves (exemple des femmes emprisonnées pour infanticide)" [membres d'associations professionnelles].

"Pour les jeunes qui ne sont pas mariés, je ne suis pas contre, l'évidence c'est que aujourd'bui les rapports sexuels sont banalisés même si on est dans un pays musulman. Ce serait bypocrite de se taire ... Je préfére qu'ils utilisent la CU plutôt que de faire un infanticide" [membre d'ONG].

\section{La CU peut contribuer au repositionnement de la PF}

"Nous sommes dans ce plaidoyer. Il faut aider les populations à corriger certaines erreurs. Donc il doit être connu et accepté par toute la population, et ne pas se limiter à une seule cible [les jeunes]" [manageur de programme].

Cependant, certains d'entre eux pensent que sa dispensation devrait être accompagnée d'un conseil pour les raisons suivantes:

- Meilleure ciblage des hommes du fait que la CU est un produit destiné spécifiquement aux femmes mais qui est bien connu des hommes qui en cas de besoin l'achètent pour leurs partenaires. En effet, un distributeur indiquait que "70 à $80 \%$ des demandeurs sont des hommes. C'est un produit destiné aux femmes, mais acheté par les hommes. Ils sont relativement jeunes et ils connaissent le prix" [un distributeur].

- Information des utilisatrices sur la CU qui est une méthode d'exception qui ne peut remplacer la contraception régulière car "les jeunes apparemment en font une contraception régulière" [un manageur de programme].

- Informer les jeunes que la CU ne protège pas contre les IST car "les utilisateurs de la CU sont plus enclins au libertinage sexuel et à ne pas se protéger contre les IST/VIH" [un membre d'ONG].

Les LOC émettent cependant certaines réserves quant à l'utilisation de la $\mathrm{CU}$, notamment le libertinage, les abus, la substitution aux méthodes régulières de PF, les considérations religieuses, l'absence de protection contre les IST/VIH/SIDA...

Selon un LOC: "Elle mérite d'être vulgarisée mais il faut d'abord promouvoir le préservatif pour prévenir les IST/ VIH. C'est une méthode d'appoint qui ne prévient pas du Sida" [membres associations professionnelles].

Perceptions sur les attitudes des prestataires/acceptabilité du produit par les prestataires

Pour certains LOC, il existe encore certaines réticences chez les prestataires malgré les formations reçues. Ces réticences seraient essentiellement liées à des considérations souvent personnelles.

"Chez les prestataires je pense qu'il y a quelques limites. Les sages-femmes sont un peu bloquées, elles font peut être la corrélation avec l'avortement. Peut-être aussi que c'est lié à la cible (les jeunes)" [partenaires au développement].

"Chez les prestataires d'un certain âge, ça peut poser problème car ils y voient leurs filles. Leurs réactions dépassent parfois le cadre du travail" [représentant ONG].

"En ce qui concerne les prestataires, il existe encore quelques préjugés. Ils n'ont pas encore tout à fait accepté cette méthode ... Il y a une certaine méfiance par rapport au fait que les gens peuvent en abuser. Et l'on constate que le counseling actuellement est très orienté (injection, Jadelle)" [autorité publique]. 


\section{Offre de CU}

Les avis des LOC demeurent très partagés en ce qui concerne les cibles privilégiés pour la CU:

Pour certains: "La CU doit se limiter à certaines catégories de femmes: celles ayant des grossesses à risque, femmes victimes de viol et d'agressions sexuelles, prostituées, femmes ayant des rapports sexuels occasionnels (femmes d'émigrés, adolescentes)" [un membre d'OCB].

Pour d'autres: " $\mathrm{La} C U$ doit être disponible pour toutes les catégories de femmes qui en ont besoin et tant qu'il n'y a pas de contre-indication pour la méthode" [un décideur].

Il en est de même pour les endroits où la CU devrait être offerte: si certains pensent qu'il faudrait élargir l'offre de CU au niveau d'autres types de structures sanitaires moins conventionnelles telles que les structures sanitaires au niveau des camps militaires, les Centres Conseils pour Adolescents (CCA), les Infirmeries Médicales des Ecoles (IME), les infirmeries d'établissements scolaires, les universités), d'autres, par contre, ne sont pas d'avis pour que la CU soit disponible au niveau communautaire: "Jusqu'aux postes de santé, il n'y a pas de problèmes. Pour les cases, je propose qu'on attende que l'OIP soit bien implantée, et même le personnel sanitaire à ce niveau peut en abuser" [un manageur de programme].

\section{Utilisation répétée de la CU}

La grande majorité des LOC pensent que le recours à la contraception doit être exceptionnel ; ils définissent l'utilisation répétée comme une utilisation qui dépasserait plus d'une fois dans l'année. La grande majorité des LOC affirment que l'utilisation répétée est un fait réel qui est surtout constatée au niveau des pharmacies privées. Ce fait est surtout constaté chez les jeunes selon les pharmaciens. Toutefois, l'absence de statistiques fiables ne leur permet pas de confirmer cette affirmation.

Les LOC pensent que l'utilisation répétée peut poser des problèmes, notamment:

- Portes ouvertes à certaines dérives ;

- Substitution/frein par rapport à la contraception régulière ;

- Exposition au VIH.

\section{Obstacles par Rapport à l'Acces et à l'Offre de CU}

\section{Accès à la CU}

Selon les LOC, les principaux obstacles qui pourraient se dresser à l'accès aux services de CU sont les suivants:

- Le manque d'information ;

- L'attitude du prestataire (accueil, préjugés...);

- La non disponibilité du produit ;

- La gêne, honte;

- L'éloignement de la source d'approvisionnement à savoir les PPS ;

- Le coût des produits de CU qui demeure encore élevé au niveau des pharmacies ;

- Les effets secondaires liés à l'utilisation du produit ;

- La stigmatisation surtout dans une zone où le contrôle social est fort: 'Lorsque quelqu'un demande ce produit on se dit soit il a fait (acte sexuel), soit il a l'intention de faire" [un décideur] ;

- Les convictions religieuses ;

- L'incompréhension du mari ;

- Les rumeurs sur les contraceptifs en général.

\section{Obstacles par rapport à l'offre}

Selon les LOC, les principaux obstacles qui pourraient se dresser à l'offre de CU sont les suivants:

- Le manque de formation des prestataires;

- Les convictions religieuses ;

- La non disponibilité du produit du fait de la péremption du stock antérieur et du non 
renouvellement du stock au niveau des PPS $^{7}$;

- Le temps d'attente jugé très long pour les clients ;

- Les formalités administratives subies par les clients;

- Les horaires de fonctionnement des structures non adaptés ;

- Le manque de confidentialité et de l'anonymat lors de la consultation (enregistrement des informations personnelles telles que le nom, l'adresse...) au niveau des structures publiques ;

- L'absence de counseling au niveau des pharmacies où l'interaction client-prestataire est très faible (absence d'espace de counseling).

Selon les LOC, les principaux défis qui pourraient se poser dans la perspective de la promotion de la CU sont les suivants:

- L'accès à l'information/communication autour du produit ;

- La disponibilité du produit ;

- La formation des prestataires y compris des pharmaciens et agents de comptoir ;

- L'accessibilité géographique et financière ;

- L'adhésion des populations et de certains prestataires ;

- L'harmonisation des pratiques sur les délais d'utilisation car les pharmacies se fient toujours à la notice ;

- La révision de la réglementation (produit du tableau A); il serait donc nécessaire de déclassifier ce produit pour faciliter son accès ;

- La qualité de l'offre (bon counseling, confidentialité, anonymat, absence de stigmatisation).

\section{Recommandations des LOC pour la Promotion de la CU}

Selon les LOC, la promotion de la CU devrait passer par une approche multisectorielle qui engagerait divers acteurs, à savoir:

Les autorités publiques (Ministère de la santé, Ministère de l'Education Nationale, Ministère de la Justice, Ministère de la Famille, Ministère de la Jeunesse...):

- Les acteurs communautaires (OCB, Relais/Pairs Educateurs, Badienu Gox) ;

- Les religieux ;

- Les parlementaires ;

- Les médias ;

- Les juristes ;

- La société civile ;

- Les communicateurs traditionnels.

Les LOC ont formulé les recommandations suivantes pour d'axes d'interventions prioritaires pour la CU:

- Renforcer l'information pour une diffusion plus large du produit ;

- Former les prestataires;

- Assurer une bonne gestion de la logistique pour garantir la disponibilité du produit ;

- Accroître l'accessibilité financière ;

- Développer le partenariat à travers une plus grande implication des pharmaciens ;

- Développer le plaidoyer auprès des décideurs ;

- Développer la recherche sur l'acceptabilité, l’innocuité des produits.

7 Le stock antérieur de Pregnon mis en place par la DSR est arrivé en péremption en septembre 2010 et ce stock. n'a été renowvelé que récemment. Ce stocke ne permet cependant pas de couvrir les besoins annuels des régions. Cette absence de disponibilité des produits de la CU se situe dans un contexte global de rupture des produits contraceptifs dû au problème de rétention de l'information sanitaire qui se traduit par la non transmission des registres trimestriels de stocks qui constituent des outils de planification des besoins contraceptifs pour les districts. 


\section{Formation des Prestataires sur la CU}

Le counseling constitue un volet important de l'offre de service qui garantit le choix éclairé du client et, par conséquent, la qualité des services offerts. Le graphique un indique que près du tiers des prestataires n'a pas reçu une formation dans le domaine du counseling PF. Ce déficit de formation est surtout noté chez les prestataires au niveau des pharmacies ; seul 13\% des agents de comptoir et $42 \%$ des pharmaciens ont bénéficié d'une formation en counseling PF. Ce pourcentage est de 94 chez les sages-femmes. Cette situation serait due au fait que les programmes de formation en PF avaient surtout ciblé les prestataires du secteur public ; le secteur privé a peu bénéficié des programmes de formation dans le domaine de la PF. Il importe donc de mieux cibler ce secteur en vue d'une meilleure standardisation de l'offre de service $\mathrm{PF}$ au plan national.

Graphique 1: Proportion de prestataires ayant déjà reçu une formation en counseling PF dans le passé selon la qualification

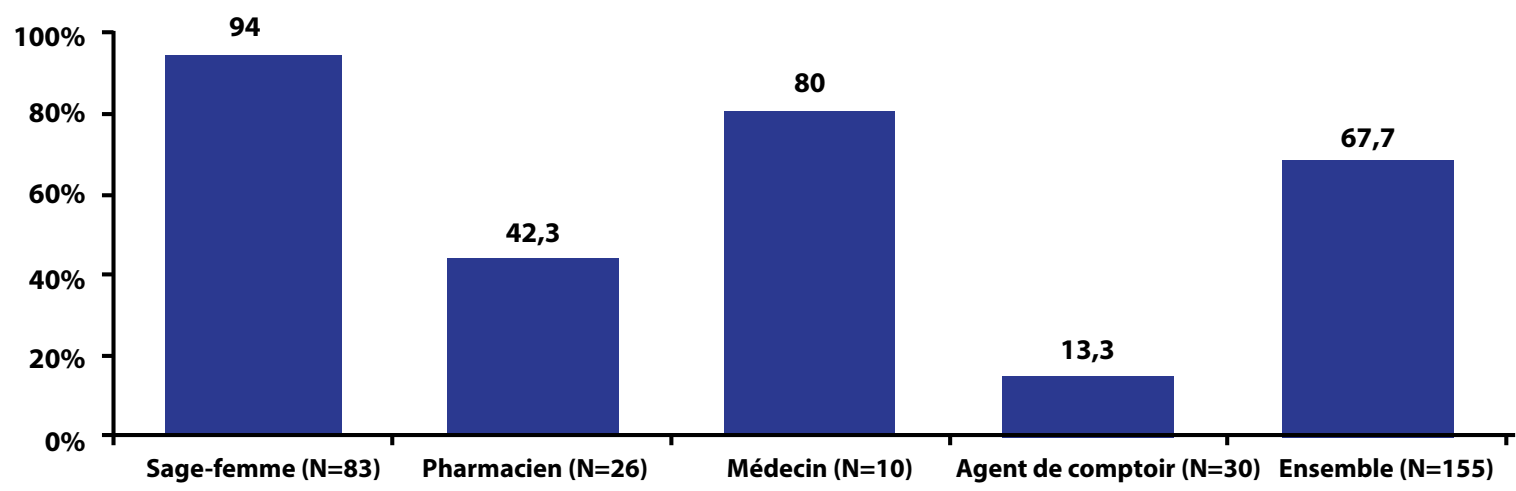

Le tableau 8 indique que la formation en CU est peu intégrée au cours de la formation initiale des prestataires. Seuls $22,6 \%$ des prestataires ont bénéficié d'une formation en CU durant leur formation de base. En effet, l'intégration de la CU dans le curriculum de formation des prestataires est très récente. Par contre, $51,6 \%$ d'entre eux l'ont reçu en cours d'emploi. On note que 38,1\% des prestataires n'ont jamais reçu de formation spécifique sur la $\mathrm{CU}$, soit près de quatre prestataires sur 10. Notons qu'à Dakar, on observe le plus fort taux de prestataires n'ayant jamais reçu de formation spécifique sur la CU $(39,5 \%)$.

Tableau 8: Pourcentage de prestataires ayant reçu une formation en CU

\begin{tabular}{|c|c|c|c|c|c|c|c|c|}
\hline \multirow[t]{2}{*}{ Formation } & \multicolumn{2}{|c|}{$\begin{array}{c}\text { Dakar } \\
(\mathrm{N}=129)\end{array}$} & \multicolumn{2}{|c|}{ Thiès $(\mathrm{N}=16)$} & \multicolumn{2}{|c|}{$\begin{array}{l}\text { Mbour } \\
(\mathrm{N}=10)\end{array}$} & \multicolumn{2}{|c|}{$\begin{array}{l}\text { Ensemble } \\
(\mathrm{N}=155)\end{array}$} \\
\hline & $\mathbf{N}$ & $\%$ & $\mathbf{N}$ & $\%$ & $\mathbf{N}$ & $\%$ & $\mathbf{N}$ & $\%$ \\
\hline $\begin{array}{l}\text { A reçu une formation spécifique en CU durant la formation } \\
\text { de base }\end{array}$ & 26 & 20.2 & 4 & 25 & 5 & 50 & 35 & 22.6 \\
\hline A reçu une formation spécifique en CU en cours d'emploi & 65 & 50.4 & 10 & 62,5 & 5 & 50 & 80 & 51.6 \\
\hline N'a pas reçu de formation spécifique sur la CU & 51 & 39.5 & 3 & 18,8 & 2 & 20 & 56 & 36.1 \\
\hline A reçu une formation en $\mathrm{CU}$ au cours des 12 derniers mois & 18 & 14 & 6 & 37,5 & 1 & 10 & 25 & 16.1 \\
\hline
\end{tabular}

Seuls $16,1 \%$ ont eu à bénéficier d'une formation spécifique sur la CU durant les 12 derniers mois ayant précédé l'enquête.

Tableau 9: Pourcentage de prestataires ayant reçu une formation en CU selon la catégorie professionnelle

\begin{tabular}{|l|c|c|c|c|c|c|c|c|}
\hline \multirow{2}{*}{ Catégorie professionnelle } & \multicolumn{2}{|c|}{ Dakar } & \multicolumn{2}{|c|}{ Thiès } & \multicolumn{2}{c|}{ Mbour } & \multicolumn{2}{c|}{ Ensemble } \\
\cline { 2 - 10 } & $\mathbf{N}$ & $\mathbf{0}$ & $\mathbf{N}$ & $\mathbf{\%}$ & $\mathbf{N}$ & $\mathbf{\%}$ & $\mathbf{N}$ & $\mathbf{\%}$ \\
\hline Sage-femme & 51 & 75,0 & 7 & 70,0 & 3 & 60,0 & 61 & 73,5 \\
\hline Pharmacien & 12 & 57,1 & 3 & 100 & 2 & 100 & 17 & 65,4 \\
\hline Médecin & 5 & 62,5 & 1 & 100 & 1 & 100 & 7 & 70,0 \\
\hline Agent de comptoir & 7 & 25,0 & 2 & 100 & & & 9 & 30,0 \\
\hline Autre & 3 & 75,0 & & & 2 & 100 & 5 & 83,3 \\
\hline Total & $\mathbf{7 8}$ & $\mathbf{6 0 , 5}$ & $\mathbf{1 3}$ & $\mathbf{8 1 , 3}$ & $\mathbf{8}$ & $\mathbf{8 0 , 0}$ & $\mathbf{9 9}$ & $\mathbf{6 3 , 9}$ \\
\hline
\end{tabular}

$63,9 \%$ des prestataires ayant déjà fourni la CU ont reçu une formation spécifique sur la CU. Les pourcentages les plus élevés de prestataires n'ayant pas reçu de formation en CU sont observés chez les agents de comptoir et les pharmaciens ( $70 \%$ et $34,6 \%$ respectivement). 


\section{Connaissance de la CU}

Le tableau 11 indique qu'il existe un pourcentage non négligeable de prestataires qui ne connaissent pas de façon précise le mode d'action de la CU ; $57,4 \%$ ont cité le blocage de l'ovulation et $63,9 \%$ l'empêchement de l'implantation de l'œuf (la nidation).

Tableau 10: Pourcentage de prestataires connaissant les modes d'action de la CU

\begin{tabular}{|l|c|c|c|c|c|c|}
\hline \multirow{2}{*}{} & \multicolumn{2}{|c|}{ Sans formation } & \multicolumn{2}{|c|}{ Avec formation } & \multicolumn{2}{c|}{ Total } \\
\cline { 2 - 7 } & $\mathbf{N}$ & $\mathbf{\%}$ & $\mathbf{N}$ & $\mathbf{\%}$ & $\mathbf{N}$ & $\mathbf{\%}$ \\
\hline Inhibe l'ovulation & 25 & 44.6 & 64 & 64.6 & 89 & $\mathbf{5 7 . 4}$ \\
\hline Empêche l'implantation & 31 & 55.4 & 68 & 68.7 & 99 & $\mathbf{6 3 . 9}$ \\
\hline Induise l'avortement & 1 & 1,8 & 1 & 1 & 2 & $\mathbf{1 , 3}$ \\
\hline Tue les spermatozoïdes & 9 & 16.1 & 19 & 19.2 & 28 & $\mathbf{1 8 . 1}$ \\
\hline Ne sait pas & 10 & 17.9 & 7 & 7.1 & 17 & $\mathbf{1 1}$ \\
\hline Autre & 6 & 10.7 & 17 & 17.2 & 23 & $\mathbf{1 4 , 8}$ \\
\hline Le délai d'utilisation de la CU est de 120 heures & 11 & 19.6 & 42 & 42.4 & 53 & $\mathbf{3 4 . 2}$ \\
\hline
\end{tabular}

On observe des écarts dans la connaissance du mode d'action de la CU entre les prestataires formés et ceux n'ayant pas reçu une formation. $44,6 \%$ des prestataires non formés ont cité le blocage de l'ovulation contre $64,6 \%$ pour les prestataires formés. On observe par ailleurs que peu de prestataires sont informés sur les dernières mises à jour sur les délais d'utilisation de la CU qui est actuellement de 120h. Les pourcentages les plus élevés sont observés chez les médecins et les sages-femmes ( $50 \%$ et $41 \%$ respectivement). Par contre, chez les pharmaciens, le niveau de connaissance demeure faible (11,5\%). Les écarts observés entre les prestataires formés et ceux non formés persistent.

Le graphique deux révèle également qu'un nombre élevé de prestataires ne savent pas que la grossesse est critère d'exclusion pour l'utilisation. Plus de la moitié des prestataires n'ont pas cité la grossesse comme critère d'exclusion de la $\mathrm{CU}^{8}$.

Graphique 2: Connaissance des contres indications médicales pour l'utilisation de la CU

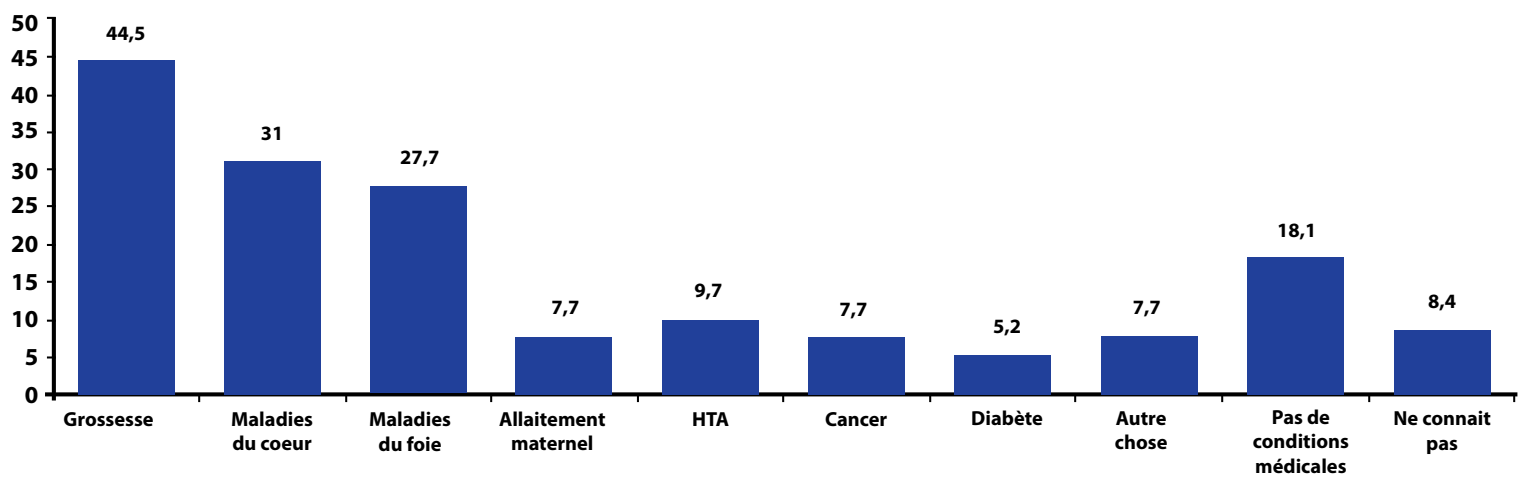

On note aussi que peu de prestataires ont cité le cancer du col comme critère d'exclusion de la CU.

Les résultats de l'étude révèle ainsi que la connaissance du mode de fonctionnement de la CU demeure encore très faible parmi les prestataires. Peu d'entre eux ont pu donner des réponses correctes quant au mode d'action de la $\mathrm{CU}$, son délai d'utilisation et les critères d'éligibilité. Il importe donc de renforcer la connaissance de ces prestataires dans ce domaine à travers l'organisation de sessions de formation.

\section{Opinions sur la CU}

Il convient de noter que peu de prestataires assimilent la pratique de la CU à une forme d'avortement. $11 \%$ d'entre eux pensent que la CU serait une forme d'avortement.

Tableau 11: Pourcentage de prestataires pensant que la CU est une forme d'avortement $(\mathrm{N}=155)$

\begin{tabular}{|l|c|c|c|c|c|c|c|c|}
\hline \multirow{2}{*}{ Catégorie professionnelle } & \multicolumn{2}{|c|}{ Dakar } & \multicolumn{2}{c|}{ Thiès } & \multicolumn{2}{c|}{ Mbour } & \multicolumn{2}{c|}{ Ensemble } \\
\cline { 2 - 9 } & $\mathbf{N}$ & $\mathbf{0}$ & $\mathbf{N}$ & $\mathbf{0}$ & $\mathbf{N}$ & $\mathbf{0}$ & $\mathbf{N}$ & $\mathbf{\%}$ \\
\hline Sage-femme & 4 & 5,9 & & & & & 4 & 4,8 \\
\hline Pharmacien & 6 & 28,6 & & & 2 & 100 & 8 & 30,8 \\
\hline Agent de comptoir & 5 & 17,9 & 1 & 50,0 & & & 6 & 20,0 \\
\hline Total & $\mathbf{1 5}$ & $\mathbf{1 1 , 6}$ & & & & & $\mathbf{1 5}$ & $\mathbf{9 , 7}$ \\
\hline
\end{tabular}

8 Les documents de formation mentionnent que la présence d'une grossesse était un critère de non éligibilité pour la CU. Les recherches ont démontré que le recours à la CU n'interférait pas sur l'évolution de la grossesse. 
Toutefois, au niveau des pharmaciens, un nombre non négligeable d'agents de comptoir $(20 \%)$ et de pharmaciens (30,8\%) l'assimilent à l'avortement. Par ailleurs, il existe encore des résistances quant à l'éligibilité de toutes les femmes à l'utilisation de la CU, contrairement aux directives nationales qui précisent que toutes les femmes sont éligibles à cette méthode, s'il n’y a pas de contre-indications médicales.

Graphique 3: Répartition des prestataires selon la connaissance des critères d'éligibilité pour la CU

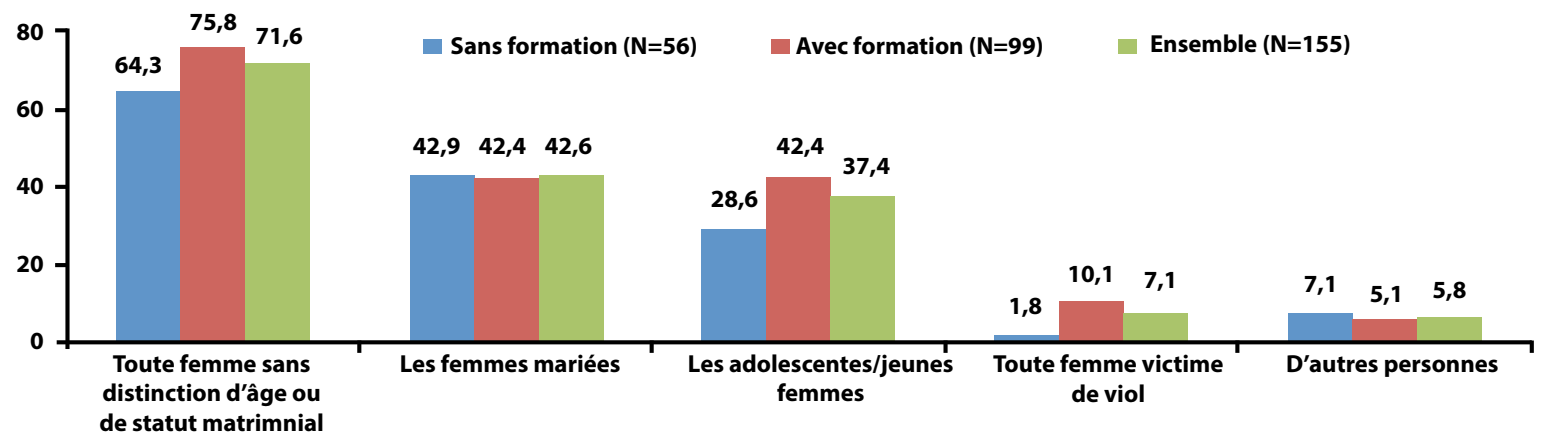

En effet, le pourcentage de prestataires ayant indiqué que toutes les femmes sans distinction de statut matrimonial étaient éligibles à la CU étaient de $75,8 \%$ et $64,3 \%$ respectivement pour les prestataires formés et ceux non formés. Moins de $45 \%$ des prestataires pensent que les femmes mariées et les adolescents étaient éligibles à la CU.

Bien que la majorité des prestataires pensent que la $\mathrm{CU}$ est un produit sûr, peu d'entre eux ont indiqué qu'elle pouvait être vendue sans ordonnance $(42,4 \%$ et $37,5 \%$ respectivement pour les prestataires formés et ceux non formés).

Graphique 4: Répartition des prestataires selon leurs opinions sur certaines conditions de l'offre de la CU (N=155)

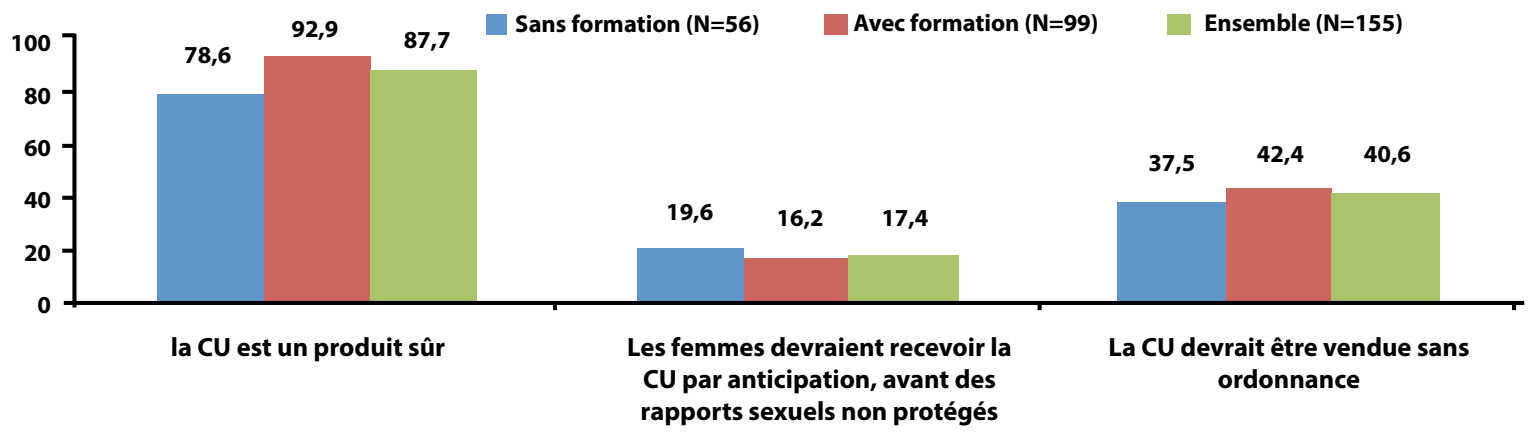

En outre, ils sont peu favorables à ce que la CU soit offerte par anticipation aux femmes avant des rapports sexuels non protégés.

Graphique 5: Répartition des prestataires selon leurs opinions sur le type de prestataire autorisé à offrir la CU

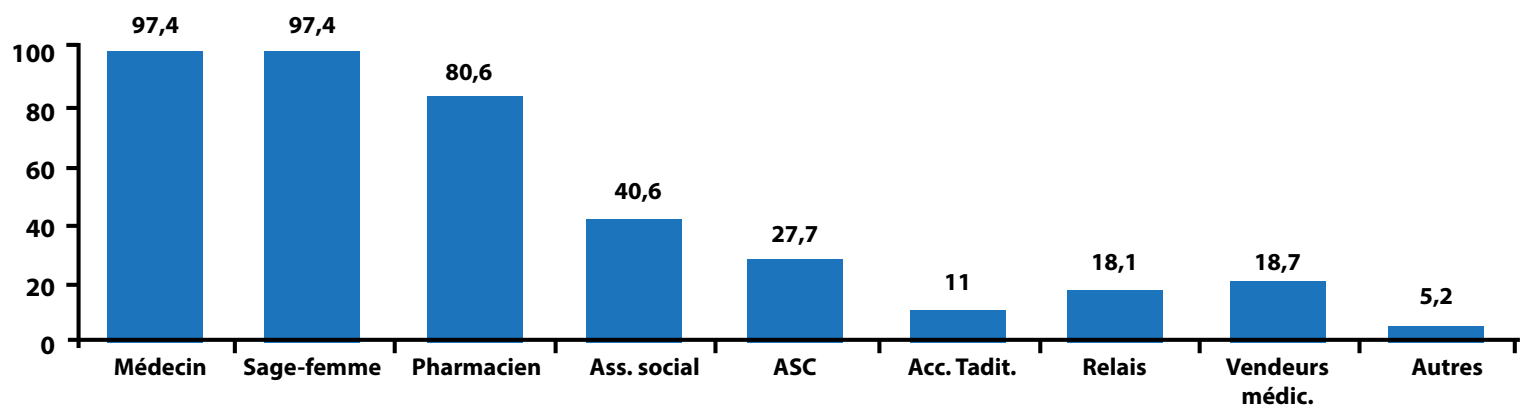

Le graphique cinq indique que si la grande majorité des prestataires est favorable à ce que la CU soit offerte par le personnel médical qualifié (médecin, sage-femme, pharmacien), la plupart sont réticents à ce que ce produit soit offert en dehors de ce type de prestataires. Seuls 40,6\% des prestataires pensent que les assistants sociaux devraient offrir la CU. En effet, le pourcentage de prestataires favorables à ce que la $\mathrm{CU}$ soit offerte par un personnel communautaire (agent de santé communautaire, relais, accoucheuses traditionnelles) demeure très faible (moins de $20 \%$ ). 
Tableau 12: Répartition des prestataires selon leurs opinions sur les endroits où la CU devrait être offerte

\begin{tabular}{|l|c|c|}
\hline \multirow{2}{*}{ Type de PPS } & \multicolumn{2}{|c|}{$\begin{array}{c}\text { Ensemble } \\
\text { (N=155) }\end{array}$} \\
\cline { 2 - 3 } & $\mathbf{N}$ & $\mathbf{\%}$ \\
\hline Hôpitaux & 148 & 95.5 \\
\hline Centres de santé & 152 & 98.1 \\
\hline Postes de santé & 147 & 94.8 \\
\hline Cases de santé & 51 & 32.9 \\
\hline Clinique privée & 126 & 81.3 \\
\hline Pharmacie & 122 & 78.7 \\
\hline Ecole & 13 & 8.4 \\
\hline Police & 6 & 3.9 \\
\hline Centre conseil Ado & 92 & 59.4 \\
\hline Autres & 11 & 7.1 \\
\hline
\end{tabular}

Ces constats sont confirmés en ce qui concerne les endroits où la CU devrait être offerte. Les opinions sont plutôt favorables pour les structures médicales classiques (hôpitaux, centres et postes de santé, cliniques privée et pharmacies) contrairement aux autres structures non classiques telles que les cases de santé, les infirmeries des écoles, la police qui pourraient renforcer l'accès aux services de CU pour certains groupes vulnérables telles que les élèves, les femmes rurales, les prisonniers. On observe qu'un pourcentage non négligeable de prestataires $(41,1 \%)$ n'est pas favorable à l'offre de la CU dans les Centres Conseils Adolescents (CCA) qui sont les endroits spécifiquement réservés à cette cible.

Graphique 6: Répartition des prestataires selon leurs opinions sur les cas où la CU devrait être utilisée

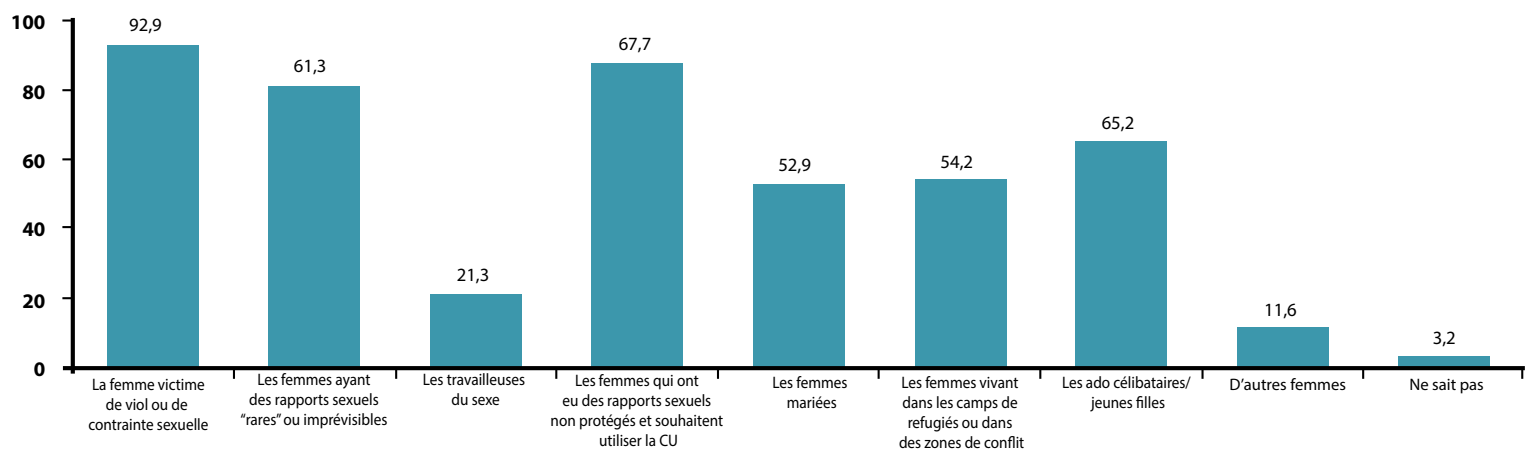

Si la plupart des prestataires sont favorables à ce que la CU soit offerte aux femmes victimes de viol et abus sexuel, aux femmes ayant des rapports sexuels non protégés et souhaitant utilisé la CU, les avis sont plus mitigés pour l'utilisation de la CU par les travailleuses du sexe, les femmes mariées, les adolescentes célibataires, les femmes vivant dans les zones de conflit (réfugiées).

\section{Perceptions surir la CU}

Les prestataires ont été interrogés pour recueillir leurs perceptions sur le profil des utilisateurs de la CU. Plus de la moitié des prestataires situerait l'âge des utilisatrices de la CU à moins de 30 ans. Près de 35\% d'entre eux indiquent que la tranche d'âge des utilisatrices de la CU se situerait entre 20 et 29 ans, $15 \%$ entre 15 et 19 ans et $4 \%$ à moins de 15 ans. Par contre, 29\% des prestataires pensent qu'elles appartiendraient à tous les âges. Toutefois, un faible pourcentage pense qu'elles seraient âgées de plus de 40 ans.

Tableau 13: Répartition des prestataires selon leurs opinions sur l'âge des utilisateurs de la CU

\begin{tabular}{|l|c|c|c|c|c|c|c|c|}
\hline \multirow{2}{*}{ Classes d'âges } & \multicolumn{2}{|c|}{ Dakar } & \multicolumn{2}{c|}{ Thiès } & \multicolumn{2}{c|}{ Mbour } & \multicolumn{2}{c|}{ Ensemble } \\
\cline { 2 - 9 } & $\mathbf{N}$ & $\mathbf{0}$ & $\mathbf{N}$ & $\mathbf{\%}$ & $\mathbf{N}$ & $\mathbf{\%}$ & $\mathbf{N}$ & $\mathbf{\%}$ \\
\hline Moins de 15 ans & 4 & 3,1 & 2 & 12,5 & & & 6 & 3,9 \\
\hline Entre 15 et 19 ans & 20 & 15,5 & 4 & 25 & & & 24 & 15,5 \\
\hline Entre 20 et 29 ans & 46 & 35,7 & 3 & 18,8 & 5 & 50 & 54 & 34,8 \\
\hline Entre 30 à et 39 ans & 16 & 12,4 & & & & & 16 & 10,3 \\
\hline 40 ans et plus & 3 & 2,3 & & & & & 3 & 1,9 \\
\hline Les femmes de tous âges & 34 & 26,4 & 6 & 37,5 & 5 & 50 & 45 & 29 \\
\hline Autre & 1 & 0,8 & & & & & 1 & 0,6 \\
\hline NSP & 5 & 3,9 & 1 & 6,3 & & & 6 & 3,9 \\
\hline Total & $\mathbf{1 2 9}$ & $\mathbf{1 0 0}$ & $\mathbf{1 6}$ & $\mathbf{1 0 0}$ & $\mathbf{1 0}$ & $\mathbf{1 0 0}$ & $\mathbf{1 5 5}$ & $\mathbf{1 0 0}$ \\
\hline
\end{tabular}

Par ailleurs, l'opinion des prestataires sur l'utilisation répétée a été abordée. La grande majorité des prestataires pense que l'utilisation de la CU devrait être limitée dans le temps. Près de $76 \%$ pensent que cela ne devrait pas excéder plus de quatre fois par an. Cela inclut près de deux prestataires sur 10 qui indiquent que la $\mathrm{CU}$ ne devrait être utilisée qu'une seule fois dans l'année et près de six prestataires sur 10 qui pensent que cela devrait se situer entre deux et quatre fois par an. Peu de prestataires pensent que la CU devrait être utilisée à chaque cycle menstruel de la femme ou à chaque fois que de besoin (moins de 2 prestataires sur 10). 
Tableau 14: Répartition des prestataires selon leurs opinions sur le nombre de fois qu'une femme peut utiliser la CU au cours de l'année

\begin{tabular}{|l|c|c|}
\hline \multirow{2}{*}{} & \multicolumn{2}{|c|}{ Ensemble } \\
\cline { 2 - 3 } & $\mathbf{N}$ & $\mathbf{\%}$ \\
\hline Une fois par an & 15 & 20.3 \\
\hline 2 à 4 fois par an & 41 & 55.4 \\
\hline 5 à 10 fois par an & 1 & 1,4 \\
\hline Une fois par mois & 6 & 8.1 \\
\hline Aussi souvent que nécessaire & 9 & 12.2 \\
\hline Autre & 1 & 1.4 \\
\hline NSP & 1 & 1.4 \\
\hline Total & $\mathbf{7 4}$ & $\mathbf{1 0 0}$ \\
\hline
\end{tabular}

On observe une perception négative des prestataires sur les femmes qui utilisent la CU. Près de $70 \%$ d'entre eux pensent qu'elles courent plus de risques d'avoir des comportements sexuels à risque, d'avoir des IST $(68 \%)$, d'avoir des partenaires sexuels multiples $(58 \%)$ et de ne pas recourir à la contraception régulière $(57 \%)$.

Tableau 15: Répartition des prestataires selon leurs perceptions des femmes qui utilisent la CU

\begin{tabular}{|l|c|c|c|c|c|c|}
\hline & \multicolumn{2}{|c|}{ Sans formation } & \multicolumn{2}{|c|}{ Avec formation } & \multicolumn{2}{c|}{ Ensemble } \\
\cline { 2 - 7 } & $\mathbf{N}$ & $\mathbf{\%}$ & $\mathbf{N}$ & $\mathbf{\%}$ & $\mathbf{N}$ & $\mathbf{\%}$ \\
\hline Plus susceptibles d'avoir des comportements sexuels à risque & 41 & 73.2 & 67 & 67.7 & 108 & 69.7 \\
\hline Plus susceptibles d'avoir des partenaires sexuels multiples & 31 & 55.4 & 59 & 59.6 & 90 & 58.1 \\
\hline Plus susceptibles d'avoir des IST & 38 & 67.9 & 67 & 67.7 & 105 & 67.7 \\
\hline $\begin{array}{l}\text { Plus susceptibles de ne pas pouvoir utiliser d'autres méthodes } \\
\text { contraceptives }\end{array}$ & 33 & 58.9 & 56 & 56.6 & 89 & 57.4 \\
\hline
\end{tabular}

\section{Offre de Services de CU}

Les données du tableau 17 indiquent que si la majorité des prestataires informe leurs clients sur le mode d'action de la CU (72\%) et son mode d'utilisation (67\%), et sur la PF (67\%), on note par contre que peu d'entre eux référent ces clients vers les services de PF (28\%), ou d'autres services de SR (29\%) ; près de la moitié $(49,7 \%)$ informe les clients sur les effets secondaires près du tiers $(32,9 \%)$ des prestataires ne donnent pas de conseils sur la PF.

Tableau 16: Répartition des prestataires selon les services habituellement offerts aux clients de la CU

\begin{tabular}{|l|c|c|}
\hline \multirow{2}{*}{} & \multicolumn{2}{|c|}{ Ensemble } \\
\cline { 2 - 3 } & $\mathrm{N}$ & $\mathbf{3}$ \\
\hline Fournit uniquement des pilules de CU & 52 & $\mathbf{3 3 , 5}$ \\
\hline Donne des informations générales sur la CU & 92 & $\mathbf{5 9 , 4}$ \\
\hline Donne des instructions sur la manière d'utiliser la CU & 104 & $\mathbf{6 7 , 1}$ \\
\hline Donne le mode d'utilisation de la CU & 112 & $\mathbf{7 2 , 3}$ \\
\hline Donne des informations sur les effets secondaires & 77 & $\mathbf{4 9 , 7}$ \\
\hline Donne des conseils sur la planification familiale & 104 & $\mathbf{6 7 , 1}$ \\
\hline Donne des informations sur les IST et le VIH & 83 & $\mathbf{5 3 , 5}$ \\
\hline Répond à leurs questions et préoccupations & 69 & $\mathbf{4 4 , 5}$ \\
\hline Réfère vers des services de planification familiale & 43 & $\mathbf{2 7 , 7}$ \\
\hline Réfère vers d'autres services & 45 & $\mathbf{2 9}$ \\
\hline Offre d'autres services aux clientes de CU & 11 & $\mathbf{7 , 1}$ \\
\hline
\end{tabular}

La grande majorité des prestataires ont indiqué qu'ils proposaient habituellement le Norlevo® à leurs clients (près de huit prestataires sur 10).

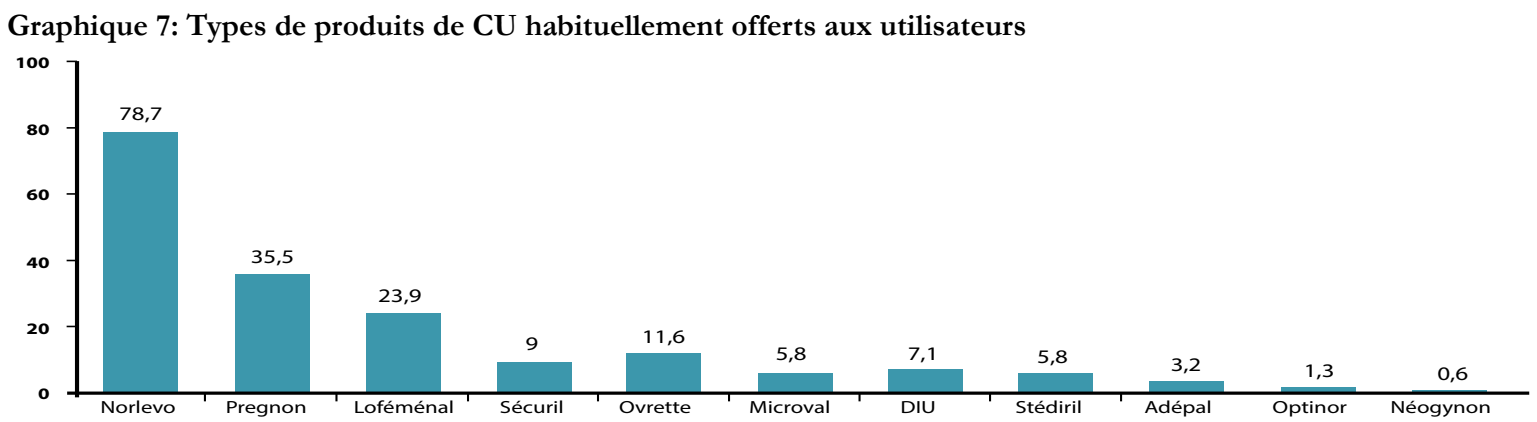

En dehors du Norlevo ${ }^{\circledR}$, le Pregnon ${ }^{\circledR}$ demeure le produit le plus proposé. Comme l'indiquent les données du graphique six, les autres produits de CU demeurent peu présents dans l'offre de CU de routine.

Selon les prestataires, les produits de CU habituellement disponibles au niveau des structures publiques, étaient surtout le Loféménal (79\%), l'ovrette (73\%), le DIU (54\%). On peut en déduire que les produits spécifiques de CU sont peu disponibles au niveau de ces structures publiques. Le Pregnon ${ }^{\circledR}$ n'a été cité que par $40 \%$ des prestataires et le Norlevo ${ }^{\circledR}$ par seulement deux $\%$ des prestataires. On peut en déduire que 
les prestataires peuvent aussi utiliser d'autres options en dehors du Pregnon. Au niveau des pharmacies privées, c'est surtout le Norlevo ${ }^{\circledR}$ qui est cité ( $98 \%$ des prestataires). Toutefois, d'autres produits tels que le Sécuril, le Microval sont disponibles. On note une présence plus variée de produits CU dans les cliniques privées comme l'indiquent les données du graphique sept, ce qui indique la possibilité de plusieurs options quant à l'offre de CU. Les autres produits sont peu présents.

Graphique 8: Type de produits disponibles selon le type de PPS
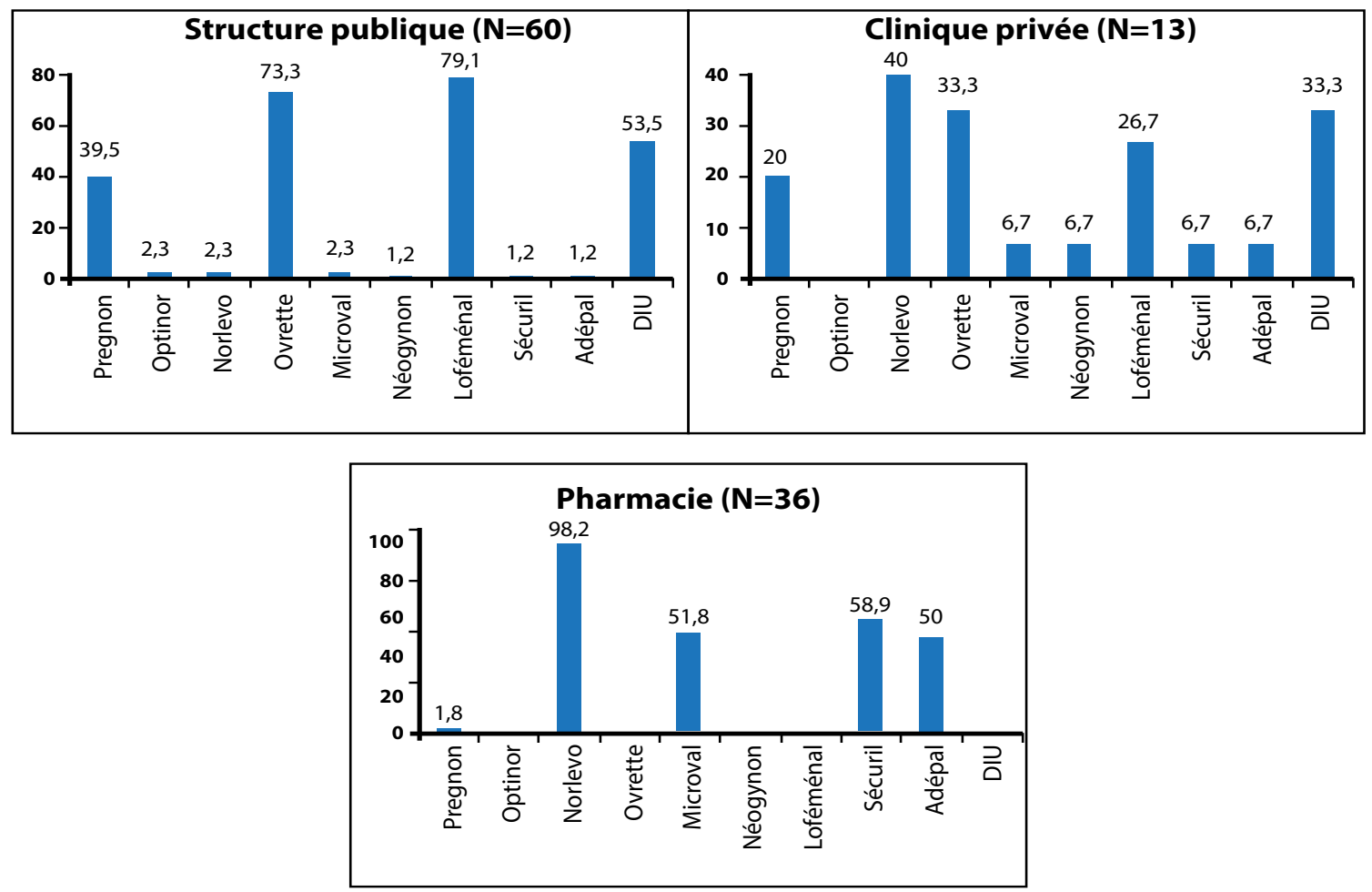

\section{Conclusion}

Les résultats des enquêtes auprès des Leaders d'Opinion Clés (LOC) et des prestataires révèlent que la CU fait partie intégrante du programme national de PF. En effet, la CU figure en bonne place aussi bien dans les documents de politiques, normes et protocoles en matière d'offre de services de SR/PF que dans les documents de formation des prestataires. Aussi bien les LOC que les prestataires sont conscients de l'importance de la CU dans la prévention des grossesses non désirées et sont favorables à l'intégration de la CU dans les directives nationales.

Toutefois, il ressort des résultats de cette étude qu'il y a un besoin urgent d'améliorer l'accessibilité et la qualité des services de CU. La grande majorité des LOC et des prestataires jugent que la sensibilisation des communautés et la vulgarisation du produit devraient constituer les activités prioritaires pour renforcer l'information des populations, des jeunes en particulier, sur le produit. Cette information des communautés pourraient ainsi contribuer à atténuer les préjugés, les inquiétudes et les réticences sur l'utilisation de la CU.

La persistance de ces préjugés autour de la CU serait liée à l'histoire récente de la CU au Sénégal. L'introduction officielle dans le système national remonte à moins de six ans. Les idées et les expériences sont actuellement en cours de construction et il faut du temps pour changer les idées et les comportements. L'histoire de la CU au Sénégal est largement tributaire de l'histoire de la PF caractérisée par une faible utilisation des méthodes modernes et par l'ampleur des pesanteurs socioculturelles. Toutefois, les perspectives d'une meilleure acceptation du produit sont ouvertes. En effet, devant l'ampleur des violences/abus sexuels, des avortements provoqués, de la question du droit à la santé des femmes et des adolescents, on observe de plus en plus une adhésion des décideurs, des communautés pour préserver la santé et le bien-être de certains groupes vulnérables que sont les adolescents/jeunes, les femmes. Ces éléments pourraient constituer une portée d'entrée légitime pour la promotion de la CU. 


\section{Recommandations}

Les principales recommandations issues de l'étude ont porté sur:

- L'information des communautés pour une diffusion plus large du produit ;

- La formation des prestataires ;

- La mise en place d'un système de gestion de la logistique pour garantir la disponibilité du produit ;

- L'amélioration de l'accessibilité financière du produit;

- Le développement du partenariat, notamment par une plus grande implication des pharmaciens ;

- Le développement des activités de plaidoyer auprès des décideurs.

\section{Références}

Division de la santé de la Reproduction. Guide de formation des prestataires de santé de la reproduction sur la contraception d'urgence. Mars 2008. 45p. En collaboration avec Population Council \& ECafrique.

Division de la santé de la Reproduction. Enquête sur la contraception d'urgence dans les pharmacies. Septembre 2009. 18p. En collaboration avec Population Council \& ECafrique.

International Consortium for Emergency Contraception. La pilule contraceptive d'urgence. Mécanisme d'action. Mai 2011. 3p.

Population Council. Rapport du forum national sur la contraception d'urgence. Novembre 2010. 15p.

Population Council. Emergency Contraception: addressing unwanted pregnancies and maternal mortality in Senegal. Fact Sheet, 2009 ; 2p. 


\section{LA CONTRACEPTION D’URGENCE}

\section{COMMENT AGIT-ELLE ? QU'EST-CE QUE C'EST ?}

Pour la pilules d'urgence (Norlévo ${ }^{\oplus}$ ):

Elle retarde l'ovulation ou la perturbe lorsquelle est sur le point de se produire

Pour le DIU d'urgence :

Au plus tard 5 jours après la date présumée de lovulation

Ne protège pas des IST

\section{L'EFFICACITÉ}

Pour le Norlévo ${ }^{\circledR}$

d'autant plus efficace si elle est prise tôt après le rapport sexuel non protégé, au plus tard dans les 120 heures ( 5 jours)

\section{Pour le stérilet :}

très grande efficacité si posé au plus tard dans les 120 heures ( 5 jours) suivant la date présumée de l'ovulation

\section{LES DIFFÉRENTES CONTRACEPTIONS D'URGENCE}

\section{La pilule d'urgence}

Norlévo :

progestatif seul - $150 \mathrm{mg}$ de Lévonorgestrel

\section{DIU d'urgence}

Le stérilet au cuivre :

Dispositif intra-utérin

\section{LES INCONVÉNIENTS}

Pour la pilule d'urgence :

En général, elles sont bien tolorées, mais des vomissements ou des nausées sont possibles. A prendre pendant un répas pour éviter les nausées. Petites douleurs de ventre

\section{COMMENT L'OBTENIR ?}

Avec ou sans prescription médicale auprès des pharmaciens

\section{LES CONTRE-INDICATIONS}

Pour Norlévo ${ }^{\circledast}$ :

Aucune 
